\title{
EFFICIENT AND DOMINANCE SOLVABLE AUCTIONS WITH INTERDEPENDENT VALUATIONS
}

\author{
Kim-Sau Chung \\ Hong Kong Baptist University, Hong Kong \\ kschung@hkbu.edu.hk \\ Jeffrey C. Ely \\ Northwestern University, U.S.A. \\ jeffelyenorthwestern.edu
}

\begin{abstract}
In auction environments in which agents have private values, the Vickrey auction induces agents to truthfully reveal their preferences and selects the efficient allocation accordingly. When the agents' valuations are interdependent, various generalizations of the Vickrey auction have been found which provide incentives for truthful revelation of all private information and preserve efficiency. However, these mechanisms generally do not provide the bidders with dominant strategies. The existing literature has therefore used a stronger equilibrium solution concept. In this paper we show that while the generalized VCG mechanism admits a multiplicity of equilibria, many of which are inefficient. We give conditions under which the efficiency equilibrium is the unique outcome of iterative elimination of ex post weakly dominated strategies. With two bidders, the standard single-crossing condition is sufficient. With more than two bidders, we show by example that a strengthening of the single-crossing condition is necessary.
\end{abstract}

Keywords: Generalized VCG mechanism, iterative elimination of ex post weakly dominated strategies.

JEL Classification Numbers: D44, D82.

This paper was first circulated in 2000. The literature has since grown much bigger than is reflected in our references. We apologize for not being able to do justice to this subsequent literature. We thank Dirk Bergemann, Eddie Dekel, Stephen Morris, and Asher Wolinsky for their continual interest in this old paper, and Zaifu Yang for inviting us to submit it to this Journal. All errors are ours.

Copyright (C) Kim-Sau Chung, Jeffrey C. Ely / 4(1), 2019, 1-38. 


\section{INTRODUCTION}

7 he Vickrey-Clarke-Groves mechanism is among the pillars of mechanism 1 design and implementation theory. In its simplest form, the Vickrey auction is a selling mechanism under which bidders with private valuations for a single object have a dominant strategy to submit a bid equal to their valuation, thereby ensuring that the auction will be won by the bidder whose valuation is the highest. More generally, in social choice environments in which agents have private values (they have all the information that is relevant to determine their preferences), and utilities are quasi-linear in money, the Vickrey-Clarke-Groves (VCG) mechanism induces agents to truthfully reveal their preferences, and selects the efficient outcome accordingly.

Attention has recently turned to efficient implementation in environments characterized by interdependent values. Values are interdependent whenever the preferences of one agent depends on some information held privately by another agent. To achieve an ex post efficient allocation in such an environment, a planner must induce truthful revelation of all private information with the understanding that the social alternative will be chosen to maximize total surplus as calculated on the basis of this information. For a simple example of such an environment, consider a planner who must decide how to allocate a single indivisible object among a given set of agents. When the agents' valuations for the object are interdependent, various generalized versions of the Vickrey auction have been found to provide incentives for truthful revelation and efficiently allocate the object. ${ }^{1}$

However, unlike in private value contexts, the generalized VCG mechanism does not provide the players with dominant-strategy incentives for truthful revelation. Instead, in these interdependent valuation contexts, the implementation notion used has been weakened to an equilibrium concept. Specifically, it has been shown that the generalized VCG mechanism admits an ex post Nash equilibrium in which all agents report truthfully. While ex post equilibrium is a more conservative equilibrium concept than, for example, Bayesian Nash equilibrium, it nevertheless presumes considerable coordination on the part of the players. In particular, there is generally a vast multiplicity of ex post

1 See Maskin (1992), Dasgupta \& Maskin (2000), Perry \& Reny (1999), Bergemann \& Välimäki (2002), Ausubel (1999), Bergemann \& Välimäki (2002), and Krishna (2003). Each of these papers, including the present one, assumes a single-dimensional type for each bidder. Jehiel \& Moldovanu (2001) prove impossibility results when types are multi-dimensional. 
equilibria, most of which are not efficient. Thus, while the existing literature has shown how the VCG mechanism can be extended to interdependent valuation settings, these generalizations have not been shown to have the same robustness as their private value counterparts.

In this paper we show some progress on this dimension. We study ex post efficient implementation in single object and multi-unit auction environments with interdependent valuations. We find conditions under which the efficient ex post equilibrium of a version of the generalized Vickrey auction is the unique outcome of iterative elimination of weakly dominated strategies. When there are two bidders and any number of identical objects, a sufficient condition for dominance solvability is a standard single-crossing condition. This condition has been observed in the literature as essentially necessary for efficient implementation even in ex post equilibrium. Thus, when there are two bidders, the generalized Vickrey auction is dominance solvable (essentially) whenever it has an efficient ex post equilibrium.

When there are more than two bidders, dominance solvability becomes a strictly stronger requirement than implementation in ex post equilibrium. We demonstrate this in the context of a symmetric linear model in which the necessary and sufficient conditions can be easily identified and interpreted. While the single crossing condition assumes only that the valuation of bidder $i$ is more sensitive to the private information of bider $i$ than that of other bidders, the necessary and sufficient condition we identify is that the influence of $i$ 's private type on his own valuation is greater than the influence of the sum of the types of the other bidders.

Interestingly however, even when our condition is not satisfied, our negative result for the case of 3 or more bidders does not conclusively rule out the existence of a dominance solvable efficient mechanism. This is because our condition applies to the class of generalized VCG mechanisms; and while it is known that all ex post efficient direct revelation mechanisms belong to this class, as we show in this paper, the revelation principle fails for our solution concept of iterative elimination of weakly dominated strategies. Thus, unlike nearly all implementation notions studied in the literature, it does not suffice for our solution concept to focus on direct revelation mechanisms. The possibility is therefore open that a dominance solvable, efficient indirect auction mechanism can be found when our condition fails.

Finally, it is well known that the outcome of iterative elimination of weakly dominated strategies often depends on the chosen order in which strategies 
are eliminated. We wish to show that the efficient ex post equilibrium is the outcome under any possible order of elimination. However, we argue by examples that in games such as auctions, order independent elimination is too much to ask for due simply to technical issues related to the infinity of strategies and payoff discontinuities. We therefore identify a weak restriction, which we call vigilantness, on the elimination procedures considered and prove that every order of elimination satisfying this restriction yields the efficient ex post equilibrium. In finite games, vigilantness reduces to the condition that the elimination procedure does not leave any dominated strategies in the ultimate solution. Thus, in finite versions of our auction environment, this latter condition is sufficient to ensure that the (finite) generalized VCG mechanism yields the efficient ex post equilibrium as the unique (i.e. order independent) dominance solution.

Dominance solvability of interdependent value auctions has been previously investigated by Harstad \& Levin (1985). These authors study affiliated pure common value auctions with a special information structure: the highest among all bidders' signals is a sufficient statistic for the full profile of signals. They show that these auctions are interim dominance-solavle.

This paper is organized as follows. In section 2 we describe the class of environments we study, and we present the versions of weak dominance, and iterative elimination of weakly dominated strategies that we employ. The concept of vigilance is defined and discussed here. In section 3, we prove our result for the case of two bidders and a single object. In section 4, we show that using a (new) version of the generalized VCG mechanism, this result can be extended to the case of two bidders and any number of identical objects. In Section 5, we study the general case in which there are more than two bidders. In a model with symmetric, linear valuation functions, we identify necessary and sufficient conditions for the generalized VCG mechanism to be dominance solvable. Finally, in section 6 we present our counterexample to the revelation principle.

\section{PRELIMINARIES}

\subsection{Dominance Solvability}

For this subsection, we consider an arbitrary game of incomplete information. Each player $i \in\{1, \ldots, n\}$ has a set $T_{i}$ of types, and a set $A_{i}$ of actions. The 
payoff to player $i$ is $\pi_{i}(a, s)$ when the type profile is $s \in T$ and the profile of chosen actions is $a \in A$. A pure strategy for player $i$ is a map $\sigma_{i}: T_{i} \rightarrow A_{i}$ specifying an action for each possible type. Let $\Sigma_{i}$ be the set of all pure strategies for $i$.

Definition 2.1. Let $\hat{\Sigma}_{-i} \subset \Sigma_{-i}$ be a subset of strategy profiles for the opponents of $i$. Strategy $\sigma_{i} \in \Sigma_{i}$ ex post weakly dominates strategy $\hat{\sigma}_{i}$ against $\hat{\Sigma}_{-i}$ if for every type profile $s \in T$ and every $\sigma_{-i} \in \hat{\Sigma}_{-i}$,

$$
\pi_{i}\left(\sigma_{i}\left(s_{i}\right), \sigma_{-i}\left(s_{-i}\right), s\right) \geq \pi_{i}\left(\hat{\sigma}_{i}\left(s_{i}\right), \sigma_{-i}\left(s_{-i}\right), s\right)
$$

with strict inequality for at least one $\sigma_{-i} \in \hat{\Sigma}_{-i}$ and $s$.

Ex-post weak dominance is the most conservative version of weak dominance possible in this incomplete-information setup. Other notions of dominance, such as ex-ante, or interim dominance, evaluate expected payoffs using some exogenous beliefs about the distribution of types. For any given belief, a strategy may be dominated in either of these senses without being ex-post dominated. On the other hand in finite games, if a strategy is ex-post dominated, then it is ex-ante and interim dominated for any full-support prior beliefs. ${ }^{2}$

In what follows, we will often say that an action $a_{i}$ is dominated for a type $s_{i}$ by another action $\hat{a}_{i}$ against a set of strategies. By this we mean that the payoff to $s_{i}$ using $\hat{a}_{i}$ is at least as high as the payoff from using $a_{i}$ and strictly higher in at least one possible case. Clearly a strategy $\sigma_{i}$ is dominated if and only if $\sigma_{i}\left(s_{i}\right)$ is dominated for at least one $s_{i}$. If we say that a strategy $\sigma_{i}$ is dominated within a given subset $\beta$ of strategy profiles, we mean that $\sigma_{i} \in \beta_{i}$ and there is an element of $\beta_{i}$ which dominates $\sigma_{i}$ against $\beta_{-i}$. Finally, we say that a set $\beta$ of strategy profiles is internally undominated if there is no strategy $\sigma_{i} \in \beta_{i}$ which is dominated within $\beta$.

In private value settings, the Vickrey auction implements the efficient allocation in dominant strategies. In general interdependent value settings, there is no efficient mechanism in which the players have a dominant strategy.

2 The finite/full-support qualification is necessary here because otherwise an ex-post dominated strategy may satisfy the strict inequality in the definition only for a profile of types which have probability zero, in which case the strategy would not be dominated in the other senses. Of course our auction mechanisms have infinite strategy sets as an efficient auction in our setting must. It is nevertheless true in each of the dominance arguments we present that the strict inequality would be satisfied with positive probability under any full-support prior. We however do not take the time to prove this in each case below. 
However, we will show below that efficiency can be achieved as the unique outcome of iterative elimination of ex-post weakly dominated strategies.

There are subtle aspects of iterative elimination of weakly dominated strategies, especially in games such as auctions in which there are infinitely many types and actions and in which payoffs are not continuous. In the remainder of this section, we formalize the types of elimination procedures we will consider.

In any game with finitely many strategy profiles $\Sigma$, an elimination sequence is a sequence $\beta^{k}$ of strategy profiles satisfying the following four conditions

1. $\beta^{0}=\Sigma$

2. $\beta^{k} \subset \beta^{k-1}$

3. $\sigma_{i} \in \beta_{i}^{k-1} \backslash \beta_{i}^{k}$ only if $\sigma_{i}$ is dominated against $\beta_{-i}^{k-1}$

4. $\cap_{k} \beta^{k}$ is internally undominated.

It is well-known that even in finite games, the outcome of iterative elimination of weakly dominated strategies can depend on the chosen order in which strategies are eliminated. Our definition of an elimination sequence imposes no requirements on the order of elimination.

Our main focus is on environments with a continuum of types, in which an efficient mechanism must have infinitely many strategies. ${ }^{3}$ In games with infinitely many strategies, the definition of an elimination sequence must be modified in two ways. First, we must strengthen the third requirement as follows.

$3^{\prime} . \sigma_{i} \in \beta_{i}^{k-1} \backslash \beta_{i}^{k}$ only if $\sigma_{i}$ is dominated against $\beta_{-i}^{k-1}$ by an element of $\beta_{i}^{k}$.

That is, we add the requirement that eliminated strategies must be dominated by a strategy that survives. In games with infinite strategy sets and discontinuous payoffs, it is possible that each element of an infinite subset of strategies is dominated by another element of the same set, without any element of the set dominated by an undominated strategy. In our positive results below, when we

3 Even if there are truly only finitely many types, the finite generalized VCG mechanism requires that the mechanism designer know precisely the set of possible types of each player. On the other hand, when there are two bidders, the second price auction can be used no matter what the size of the set of types. So if we are interested in mechanisms which minimize the informational demands on the designer, we are forced to consider infinite mechanisms. 
construct an elimination sequence leading to the efficient equilibrium, we will be careful to show that all eliminated strategies are dominated by strategies that are not themselves dominated.

We wish also to show that the solutions we obtain by our elimination sequences would be obtained under any alternative order of elimination. However, when the mechanism has infinitely many strategies, the definition of an elimination sequence for finite games is far too permissive. We will modify ${ }^{4}$ condition 4 as follows. Let $\mathscr{D}^{k}$ be the set of strategies that are dominated within $\beta^{k-1}$ by an element of $\beta^{k}$.

$4^{\prime}$. There exists a $K$ such that if $\sigma_{i} \in \mathscr{D}^{k}$ for $K$ consecutive rounds $k=$ $k_{1}, \ldots k_{K}$, then $\sigma_{i} \notin \beta^{k_{K}+1}$.

In finite games, condition $4^{\prime}$, which we call vigilance, is equivalent to 4 . In infinite games, it is possible to construct pathological elimination sequences in which a strategy is dominated in every round of the process, but if not eliminated in any finite stage, is undominated within the final set of strategy profiles. Vigilance rules out such elimination sequences. Another possibility is that a set of strategies $X$ can be dominated in every round, and if they are eliminated at any finite stage then a further set of strategies $Y$ could be eliminated. With an invigilant elimination sequence it is possible that the set $X$ is eliminated only "at the limit," at which point it is no longer possible to eliminate $Y{ }^{5}$ Vigilance will ensure that all strategies in $X$ are eliminated

4 Condition $4^{\prime}$ is not a strict strengthening of condition 4 . In some games, there are elimination sequences which satisfy $1,2,3^{\prime}$, and $4^{\prime}$, but which do not satisfy 4 . In particular, it can happen that a strategy which is undominated in every round becomes dominated only in the limit set. The appropriate solution to such situations would be to extend the elimination procedure to trans-finite rounds. In our application, $4^{\prime}$ does imply 4 as this problem does not arise. We therefore do not introduce the necessary additional notation.

${ }^{5}$ Here is a two-player example illustrating both possibilities. Each player's strategies are the integers. The payoffs are as follows. If $\sigma_{i}>0$, then $\pi_{i}\left(\sigma_{i}, \sigma_{-i}\right)=1-1 / \sigma_{i}$ if $\sigma_{i} \leq \sigma_{-i}+1$ and -1 otherwise. $\pi_{i}(0, \cdot) \equiv \varepsilon>0$. For player $2, \pi_{2}\left(\cdot, \sigma_{2}\right) \equiv-1$ for $\sigma_{2}<0$, and for player 1 , when $\sigma_{1}<0, \pi_{1}\left(\sigma_{1}, \sigma_{2}\right)=1$ if $\sigma_{2}<0$, otherwise $\varepsilon$ if $\sigma_{2}=0$ and -1 if $\sigma_{2}>0$. Consider first the elimination sequence given by $\beta_{1}^{k}=\left\{\sigma_{1}: \sigma_{1} \leq 0\right.$ or $\left.\sigma_{1}>k\right\}$ and $\beta_{2}^{k}=\{0\} \cup\left\{\sigma_{2}: \sigma_{2}>k\right\}$. This elimination sequence is not vigilant because the negative strategies of player 1 are dominated in every stage after round 1, but are never eliminated. Note that the negative strategies are not dominated in the limit strategy set because none of the positive strategies remain despite the fact that for every finite $k$, there are infinitely many positive strategies in $\beta^{k}$. Hence condition 4 is satisfied in this case. Next consider the elimination sequence given by $\beta_{1}^{k}=\left\{\sigma_{1}: \sigma_{1} \leq 0\right.$ or $\left.\sigma_{1}>k\right\}$ and $\beta_{2}^{k}=\{0\} \cup\left\{\sigma_{2}:\left|\sigma_{2}\right|>k\right\}$. This elimination sequence 
in finite time. In infinite games, we define an elimination sequence to be a sequence satisfying conditions $1,2,3^{\prime}, 4^{\prime}$.

Definition 2.2. A dominance solution of an incomplete information game with strategy profiles $\Sigma$, is a subset $\beta \subset \Sigma$ such that for some elimination sequence $\beta^{k}, \beta=\cap_{k} \beta^{k}$.

We pause here to note the role that vigilance plays in our results. We present two types of results below. First, for each of our mechanisms we exhibit an efficient dominance solution. Second, we argue that every dominance solution is efficient (recall that with weak dominance, we cannot take this for granted as we could with strict dominance which is order-independent). By limiting (albeit by what we argue is a purely technical restriction) the set of elimination sequences under consideration, we strengthen the first type of result, but weaken the second.

Note that for any game, eliminating all dominated strategies in every round yields an vigilant elimination sequence and hence every game has at least one dominance solution (although it may be empty).

Finally, while we focus on the solution concept of iterative elimination of weakly dominated strategies, we now recall the definition of ex post equilibrium, the solution concept previously studied in this context.

Definition 2.3. An ex post equilibrium is a profile $\left(\hat{\Sigma}_{1}, \ldots, \hat{\Sigma}_{n}\right)$ of strategy subsets with $\hat{\Sigma}_{i} \subset \Sigma_{i}$, such that for each strategy profile $\left(\sigma_{1}, \ldots, \sigma_{n}\right)$ with $\sigma_{i} \in \hat{\Sigma}_{i}$, each type profile $s$, and each player $i$,

$$
\pi_{i}\left(\sigma_{i}\left(s_{i}\right), \sigma_{-i}\left(s_{-i}\right), s\right) \geq \pi_{i}\left(a_{i}, \sigma_{-i}\left(s_{-i}\right), s\right)
$$

for each action $a_{i} \in A_{i}$.

Our definition is non-standard because it ascribes sets of (pure) strategies to each player, rather than a unique strategy. Because the definition implies that each player $i$ is (ex post) indifferent among each of the strategies in $\hat{\Sigma}_{i}$, regardless of the strategies in $\hat{\Sigma}_{-i}$ used by the remaining players, we can interpret this as a mixed-strategy ex post equilibrium. There is no need to specify

is not vigilant because all negative strategies for player 2 are dominated in every round, but it takes infinitely many rounds to eliminate them. If they were eliminated in finite time, as required by vigilance, then the negative strategies of player 1 could also be eliminated. Again, condition 4 is satisfied. 
the randomization used by the players in this mixed-strategy equilibrium, because each player would be indifferent among all of his equilibrium strategies regardless of the mixtures used by the opponents.

\subsection{Auctions with Interdependent Valuations}

We consider auction settings with $n$ bidders competing for a single object (we will generalize this framework to multi-unit auctions in Section 4). The set of bidders is $\mathscr{I}$. Each bidder $i$ is assumed to observe a private type $s_{i} \in T_{i}=[0,1]$. Bidder $i$ 's value for the object depends on the realized profile of types $s \in T:=\prod T_{i}$ according to the continuous function $v_{i}: T \rightarrow \mathbf{R}_{+}$, where $v_{i}$ is strictly increasing and satisfies the following standard single-crossing condition:

Assumption 1 (Single-Crossing Property). For any $i \neq j$ and $\hat{s}_{-i}$, the difference

$$
g\left(s_{i}\right)=v_{i}\left(s_{i}, \hat{s}_{-i}\right)-v_{j}\left(s_{i}, \hat{s}_{-i}\right)
$$

crosses zero at most once, and from below.

When bidder $i$ is awarded the object and makes payment $t_{i}$, his net payoff is $v_{i}(s)-t_{i}$.

We will consider direct and indirect auction mechanisms. An auction mechanism is a triple $(A, p, t):=\left(\left\{A_{i}\right\}_{i=1}^{n},\left\{p_{i}\right\}_{i=1}^{n},\left\{t_{i}\right\}_{i=1}^{n}\right)$. Here $A_{i}$ is a set of actions available to bidder $i$. In a direct mechanism, these will be reports of bidders' private types, while in an indirect mechanism, they could be bids, or more complicated messages. For each $i, p_{i}: A \rightarrow[0,1]$ is a mapping specifying the probability $p_{i}(a)$ with which $i$ is awarded the object when the profile of actions is $a$. The vector $p(a)=\left(p_{1}(a), \ldots, p_{n}(a)\right)$ is called the outcome. Finally $t_{i}: A_{i} \rightarrow \mathbf{R}$ specifies the transfer made by bidder $i$ to the auctioneer as a function of the chosen actions.

Any dominance solution $\beta$ of an auction mechanism yields an outcome correspondence $f: T \rightrightarrows \Delta\{1, \ldots, n\}$. The set $f(s)$ of possible outcomes for type profile $s$ is the set $\{p(a): a=\sigma(s)$ for some $\sigma \in \beta\}$. For any $p \in \Delta\{1, \ldots, n\}$, let $C(p)$ denote the support of $p$. The (ex post) efficient allocation correspondence is $e$, defined by $p \in e(s)$ iff $C(p) \subset \operatorname{argmax}_{i} v_{i}(s)$. (We are implicitly assuming that it is never efficient for the auctioneer to keep the object.) We will say that a dominance solution $\beta$ of an auction mechanism is efficient if its outcome correspondence satisfies $\emptyset \neq f(s) \subset e(s)$ for every $s$. 
Definition 2.4. The efficient allocation correspondence is ex post dominance implementable if there exists a mechanism of which every dominance solution is an efficient ex post equilibrium.

Note that our implementation notion requires that the dominance solution also be an ex post equilibrium. Another potentially problematic feature of iterative elimination of dominated strategies in infinite games is that the dominance solution in general need not be a Nash equilibrium. In such a case, we would obviously lose faith in our proposed dominance solution, and this motivates the additional requirement. Fortunately, as we show, the generalized VCG mechanism does not suffer from this problem.

\section{AUCTIONS WITH TWO BIDDERS}

This section considers the case of two bidders and one object. We shall prove that the single crossing property is sufficient for efficient allocation to be ex post dominance implementable.

Theorem 3.1. When there are two bidders, the efficient allocation is ex post dominance implementable.

\subsection{Illustration of the Proof}

The proof of Theorem 3.1 is lengthy primarily because of the complications introduced by asymmetric valuation functions. However, the main idea can be understood with a simple diagram in the special case in which $v_{1}(0,0)=$ $v_{2}(0,0)$ and $v_{1}(1,1)=v_{2}(1,1)$, i.e. symmetry at the extremes. We normalize these extreme values to 0 and 1 respectively.

In figure 1 we have depicted a box representing the set of type profiles in a two-bidder auction environment. The horizontal and vertical axes are $s_{1}$ and $s_{2}$ respectively. Our assumptions of continuity, monotonicity and single crossing imply that there is a continuous curve, which we will refer to as the "pivot curve" consisting of all type profiles in which the bidders' valuations are equal. In the special case we analyze here, the pivot curve connects the bottom left corner with the upper right corner of the box. By the single crossing condition, everywhere to the right of this curve, efficiency demands that the object be awarded to bidder 1 , and to the left, bidder 2 . 


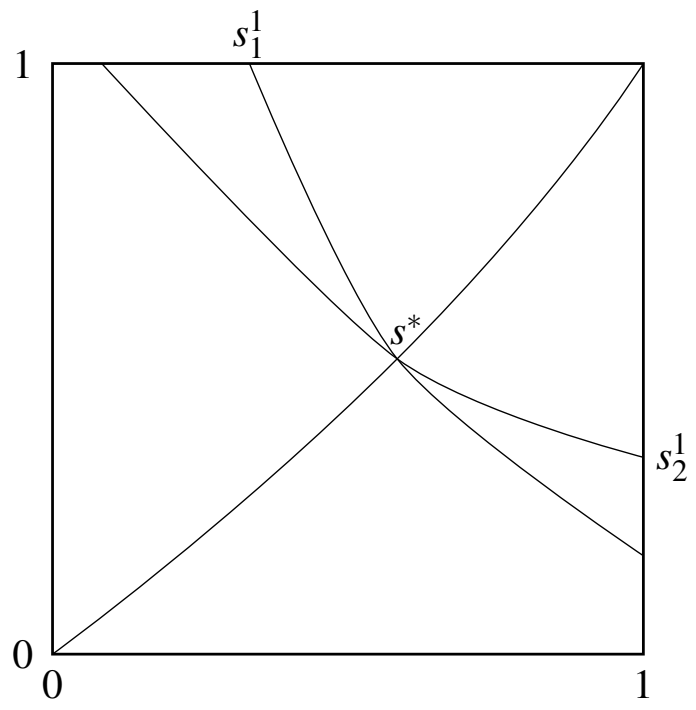

Figure 1: Pivot Curve and Indifference Curves

For every point $s$ on the pivot curve, let $v(s)$ denote the bidders' common valuation for the object at type profile $s$. By monotonicity, the value $v(s)$ increases as $s$ moves up the pivot curve. In a standard second-price auction, the following strategies constitute an ex post equilibrium (see Maskin (1992)). When bidder $i$ has type $s_{i}$, he bids the value $v\left(s_{i}, s_{-i}\right)$, where $\left(s_{i}, s_{-i}\right)$ is the point on the pivot curve whose $i$ th coordinate is $s_{i}$. That is, bidder $i$ bids his pivotal valuation, which we denote by $b_{i}\left(s_{i}\right)$. Since this bidding behavior implies that 1 outbids 2 if and only if $s$ is to the right of the pivot curve, this equilibrium yields an ex-post efficient allocation.

Unfortunately, this efficient equilibrium is not the unique ex post equilibrium of the second-price auction. In fact, there are uncountably many inefficient ex post equilibria. A simple class of inefficient ex post equilibria is constructed as follows. ${ }^{6}$ Fix any $0<\Delta \leq 1 / 2$. Modify the efficient bidding strategies so that each type $s_{1}$ such that $b_{1}\left(s_{1}\right) \in[1 / 2-\Delta, 1 / 2+\Delta]$ bids

6 The following inefficient ex post equilibria translate naturally to inefficient undominated ex post equilibria of the English auction. Krishna (2003) provides examples of inefficient perfect Bayesian equilibria of the English auction, but his examples are either dominated or fail the "ex post regret" criterion. Applying our iterative dominance arguments to ascending auctions with interdependent valuations is left for future work. 
$v_{2}\left(s_{1}, b_{2}^{-1}(1 / 2+\Delta)\right.$, and each type $s_{2}$ such that $b_{2}\left(s_{2}\right) \in[1 / 2-\Delta, 1 / 2+\Delta]$ bids $v_{1}\left(b_{1}^{-1}\left(1 / 2-\Delta, s_{2}\right)\right.$. Other types bid as in the efficient equilibrium. It can easily be checked that this is an ex post equilibrium, and the object is misallocated whenever $1 / 2-\Delta<b_{1}\left(s_{1}\right)<b_{2}\left(s_{2}\right)<1 / 2+\Delta$.

This multiplicity may not be surprising at first; indeed even in the case of private values, there are inefficient equilibria of the second-price auction. However, with private values, all inefficient equilibria involve dominated strategies. This is not the case in our setting. When $\Delta$ is chosen sufficiently small, the strategies described above are not dominated. Furthermore, for any $k, \Delta$ can be chosen small enough that the resulting strategies are not dominated even after $k$ rounds of elimination.

We now argue, however, that for any type $s_{i}$, any bid not equal to $b_{i}\left(s_{i}\right)$ would be eliminated after sufficiently many rounds of elimination. The first step is to eliminate for all types of either bidder bids greater than 1 and less than 0 . Bids greater than 1 are dominated because the value of the object is always less than or equal to 1 , and bids below 0 are dominated because the value of the object is always greater than or equal to 0 .

Next consider a point $s^{*}$ on the pivot curve. Let $b=b_{1}\left(s_{1}^{*}\right)=b_{2}\left(s_{2}^{*}\right)$ be the value that each bidder assigns to the object when the type profile is $s^{*}$. We can find "indifference curves" for each bidder $i$ through the point $s^{*}$, connecting all of the type profiles at which bidder $i$ assigns value $b$ to the object. The single crossing condition implies that bidder 1 's indifference curve lies above that of bidder 2 to the left of the pivot curve, and below to the right, as shown in figure 1.

Consider the type $s_{1}^{1}$ of bidder 1 for whom $v_{1}\left(s_{1}^{1}, 1\right)=b$. By monotonicity, for any type $s_{1}<s_{1}^{1}$, the maximum possible value for the object, $v_{1}\left(s_{1}, 1\right)$ is strictly less than $b$. Moreover, for any such $s_{1}$, there is a bid $b^{\prime}<b$ such that type $s_{1}$ 's valuation is less than or equal to $b^{\prime}$ for any possible type of bidder 2 . By bidding $b$, type $s_{1}$ will win the auction and earn a strictly negative net payoff whenever the opponent bids between $b^{\prime}$ and $b$. By instead bidding $b^{\prime}$, $s_{1}$ will lose the auction in all these cases and obtain a payoff of zero. Since only in these cases does $b^{\prime}$ change the outcome relative to $b$, it follows that $b^{\prime}$ dominates $b$ for type $s_{1}{ }^{7}$ Thus, we can eliminate a bid of $b$ for any type of bidder 1 below $s_{1}^{1}$. By the same argument, we can eliminate $b$ for any type of bidder 2 below $s_{2}^{1}$, and we can apply the analogous arguments for all possible

7 This sketch ignores the possibility that $b^{\prime}$ itself may be eliminated. In fact, as we show in the proof, an undominated bid can be found which dominates both $b^{\prime}$ and $b$ in this case. 
bids corresponding to pivotal valuations.

Now consider type $s_{2}^{2}$, depicted in figure 2. This type is defined by $v_{2}\left(s_{1}^{1}, s_{2}^{2}\right)=b$. After having eliminated $b$ for all types of bidder 1 less than $s_{1}^{1}$, bidder 2 can deduce, whenever he wins at a price $b$, that bidder 1 's type is no less than $s_{1}^{1}$. This means in particular that conditional on winning at price $b$, the value of the object to type $s_{2}^{2}$ is at least $b$. And by monotonicity, for any type $s_{2}>s_{2}^{2}$, the object would be worth strictly more than $b$.

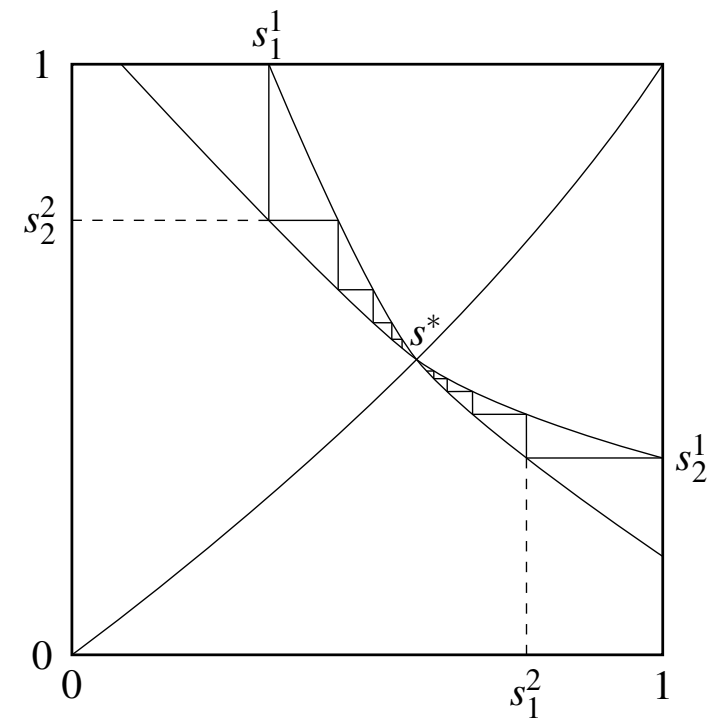

Figure 2: Iterative Elimination for $b$

We wish to conclude from this that $b$ is dominated for such a type $s_{2}$ by some $b^{\prime}>b$. To make this argument we must calculate the payoff to $s_{2}$ conditional on winning at prices slightly higher than $b$. The assumed continuity of the valuation functions implies that for bids near $b$, the set of types of bidder 1 eliminated in round one is not much different than the set eliminated for $b$, namely $\left[0, s_{1}^{1}\right)$. In particular, the smallest type that remains for a bid slightly higher than $b$ is close to $s_{1}^{1}$. This means that we can find a $b^{\prime}$ higher than, but close enough to $b$ such that conditional on winning against any bid between $b$ and $b^{\prime}$ type $s_{2}$ is ensured of a strictly positive net payoff. It follows that $b^{\prime}$, which leads to a different allocation only in these cases, dominates $b$ for $s_{2} .{ }^{8}$

8 Again, a more careful argument is required to show that $b$ is dominated by an undominated $b^{\prime}$. 
This argument shows that in the second round of elimination, $b$ can be eliminated for every type of bidder 2 greater than $s_{2}^{2}$. The analogous argument eliminates $b$ for all types of bidder 1 greater than $s_{1}^{2}$. As suggested by figure 2, we can now iterate this argument deriving bounds $s_{1}^{k}, s_{2}^{k}$ successively eliminating $b$ for more and more types of each bidder. We claim that the sequences $s_{1}^{k}$ and $s_{2}^{k}$ converge to $s_{1}^{*}$ and $s_{2}^{*}$ respectively, and thus that these are the the only types for whom $b$ is not eliminated after infinitely many rounds of elimination. Indeed, if $v_{1}\left(s_{1}^{k}, s_{2}^{k-1}\right)=v_{2}\left(s_{1}^{k}, s_{2}^{k+1}\right)=b$ for every $k$, and if $\left(s_{1}^{k}, s_{2}^{k}\right)$ were any convergent subsequence of these bounds with limit $\hat{s}=\left(\hat{s}_{1}, \hat{s}_{2}\right)$, then by continuity of the valuation functions $v_{1}(\hat{s})=v_{2}(\hat{s})=b$, i.e., $\hat{s}=s^{*}$.

Since this argument applies to every bid $v(s)$ for $s$ on the pivot curve, and since we have already eliminated all other bids for every type of both bidders, after iterative elimination of ex-post weakly dominated strategies there remains a unique bidding strategy for each bidder $i$, namely the efficient ex-post equilibrium in which $s_{i}$ bids $b_{i}\left(s_{i}\right)$.

The formal proof below treats the asymmetric case and also shows that not only this elimination sequence, but every vigilant elimination sequence leads to the efficient allocation.

\subsection{Proof of Theorem 3.1}

We consider the following generalized VCG mechanism. Each bidder $i$ 's message space is $[0,1]$. The set of reporting strategies is the set $R_{i}$ of maps $\rho_{i}:[0,1] \rightarrow[0,1]$. Given the profile of reports $r$, bidder $i$ 's imputed valuation is $v_{i}(r)$, and the object is awarded to the bidder with the highest imputed valuation. Ties are broken by randomization. The winning bidder $i$ makes a payment to the auctioneer equal to his pivotal valuation: $v_{i}\left(s_{i}^{*}, r_{-i}\right)$ where $s_{i}^{*}=\min \left\{s_{i} \in[0,1]: v_{i}\left(s_{i}, r_{-i}\right) \geq v_{-i}\left(r_{-i}, s_{i}\right)\right\}$. The loser makes no transfer. As is well-known, the truthful reporting strategies form an ex-post equilibrium of this mechanism whose outcome is ex-post efficient.

We begin by introducing some notation. Consider the set $S$ of type pairs $s$ such that $v_{1}(s)=v_{2}(s)$. If $S=\emptyset$, then by the intermediate value theorem (hereafter IVT), either $v_{1}>v_{2}$ for every type profile or $v_{2}>v_{1}$ for every type profile. In either case, every strategy profile brings about the unique efficient allocation. In particular, no strategy is dominated, $R$ is the unique dominance solution and we are done. So assume $S \neq \emptyset$, and let $S_{i} \subset[0,1]$ be the projection of this set into bidder $i$ 's set of types. 
Lemma 3.2. $1 . S_{i}$ is an interval $\left[s_{i}, \bar{s}_{i}\right]$.

2. If $s_{i}<\underline{s}_{i}$ then $v_{i}\left(s_{i}, \hat{s}_{-i}\right)<v_{-i}\left(\hat{s}_{-i}, s_{i}\right)$ for every $\hat{s}_{-i}$.

3. If $s_{i}>\bar{s}_{i}$ then $v_{i}\left(s_{i}, \hat{s}_{-i}\right)>v_{-i}\left(\hat{s}_{-i}, s_{i}\right)$ for every $\hat{s}_{-i}$.

4. If $\underline{s}_{i}>0$ then $\underline{s}_{-i}=0$.

5. If $\bar{s}_{i}<1$ then $\bar{s}_{-i}=1$.

Proof. Suppose $s_{i} \notin S_{i}$. There exists no $s_{-i} \in[0,1]$ such that $v_{i}\left(s_{i}, s_{-i}\right)=$ $v_{-i}\left(s_{-i}, s_{i}\right)$. Then either $v_{i}\left(s_{i}, s_{-i}\right)-v_{-i}\left(s_{-i}, s_{i}\right)>0$ for every $s_{-i}$, or the opposite inequality holds for every $s_{-i}$. This is a consequence of the intermediate value theorem (hereafter IVT) and the continuity of $v_{i}$ and $v_{-i}$.

The single crossing condition implies in the former case that for all $s_{i}^{\prime}>s_{i}$, and every $s_{-i}, v_{i}\left(s_{i}^{\prime}, s_{-i}\right)>v_{-i}\left(s_{-i}, s_{i}^{\prime}\right)$, and hence $s_{i}^{\prime} \notin S_{i}$. In the latter case the conclusion is that $s_{i}^{\prime} \notin S_{i}$ for every $s_{i}^{\prime}<s_{i}$. This argument shows that the complement of $S_{i}$ is of the form $\left[0, s_{i}\right) \cup\left(\bar{s}_{i}, 1\right]$, and hence that $S_{i}$ is an interval.

To prove the second part, note that $v_{i}\left(\underline{s}_{i}, \underline{s}_{-i}\right)=v_{-i}\left(\underline{s}_{-i}, s_{i}\right)$. By the singlecrossing condition $v_{i}\left(s_{i}, \underline{s}_{-i}\right)<v_{-i}\left(\underline{s}_{-i}, s_{i}\right)$, since $s_{i}<\underline{s}_{-i}$. There could not be any $\hat{s}_{-i}$ such that $v_{i}\left(s_{i}, \hat{s}_{-i}\right)>v_{-i}\left(\hat{s}_{-i}, s_{i}\right)$ otherwise the IVT would imply the existence of a $s_{-i}^{\prime}$ such that $v_{i}\left(s_{i}, s_{-i}^{\prime}\right)=v_{-i}\left(s_{-i}^{\prime}, s_{i}\right)$ which is impossible since $s_{i}<s_{i}$. The third part is proven by a similar argument.

The fourth and fifth claims follow immediately from the second and third.

In view of this result, we will write $S_{i}=\left[s_{i}, \bar{s}_{i}\right]$ (by the continuity of the valuation functions, $S_{i}$ is closed). For each $s_{i} \in \bar{S}_{i}$, we denote by $b_{i}\left(s_{i}\right)$ the unique value $b$ such that there exists a $s_{-i} \in T_{-i}$ for which $v_{i}\left(s_{i}, s_{-i}\right)=v_{-i}\left(s_{-i}, s_{i}\right)=b$. Observe that $b_{-i}\left(r_{-i}\right)$ is the generalized VCG payment bidder $i$ would have to make if he were to win the auction against a report of $r_{-i} \in S_{-i} \cdot{ }^{9}$ Furthermore $b_{1}\left(\underline{s}_{1}\right)=b_{2}\left(\underline{s}_{2}\right):=\underline{b}$ and $b_{1}\left(\bar{s}_{1}\right)=b_{2}\left(\bar{s}_{2}\right):=\bar{b}$. From the continuity and monotonicity of the valuation functions, $b_{i}(\cdot)$ is a continuous, increasing bijection between $S_{i}$ and $B:=[\underline{b}, \bar{b}]$. We now extend $b_{i}$ to all of $T_{i}=[0,1]$, by specifying $b_{i}\left(s_{i}\right)=v_{i}\left(s_{i}, 1\right)$ for $s_{i}>\bar{s}_{i}$ and $b_{i}\left(s_{i}\right)=v_{i}\left(s_{i}, 0\right)$ for $s_{i}<s_{i}$.

9 In fact, the value $b_{i}\left(s_{i}\right)$ is the bid that type $s_{i}$ would make in the efficient ex post equilibrium of the second-price auction. Thus, our arguments below that a report $r_{i}$ is dominated for a type $s_{i}$, are equivalent to showing that, in the second-price auction, the bid $b_{i}\left(r_{i}\right)$ is dominated for type $s_{i}$. 
We now introduce the following relation, which is central to the argument. Given $b \in B$, and $s_{-i} \in T_{-i}$, implicitly define $\varphi_{i}\left(s_{-i}, b\right) \in[0,1]$ by

$$
v_{i}\left(\varphi_{i}\left(s_{-i}, b\right), s_{-i}\right)=b
$$

where this exists. Note that when such a type exists, it is unique by the strict monotonicity of $v_{i}$.

Lemma 3.3. 1. $\varphi_{i}\left(s_{-i}, \cdot\right)$ is continuous on its domain of definition.

2. $\varphi_{i}(\cdot, b)$ is decreasing on its domain of definition.

3. If $s_{i}=\varphi_{i}\left(s_{-i}, b\right)$ exists, then $\varphi_{-i}\left(s_{i}, b\right)$ exists.

4. For $i=1,2$, for each $b \in B$, either $\varphi_{i}(1, b)$ or $\varphi_{-i}(0, b)$ exists.

Proof. The first two claims follow from our assumed properties of $v_{i}$ : continuity in the first case, monotonicity in the second case.

To prove the third, assume first $v_{-i}\left(s_{-i}, s_{i}\right) \leq b$. Then $v_{i}\left(s_{i}, s_{-i}\right) \geq v_{-i}\left(s_{-i}, s_{i}\right)$ and therefore $s_{i} \geq s_{i}$ by part 2 of Lemma 3.2. If $s_{i} \geq \bar{s}_{i}$, then by monotonicity $v_{-i}\left(\bar{s}_{-i}, s_{i}\right) \geq v_{-i}\left(\bar{s}_{-i}, \bar{s}_{i}\right) \geq b$. On the other hand, if $s_{i}<\bar{s}_{i}$, then there exists $\hat{s}_{-i}$ such that $v_{i}\left(s_{i}, \hat{s}_{-i}\right)=v_{-i}\left(\hat{s}_{-i}, s_{i}\right)$. By the single-crossing condition, since $v_{-i}\left(s_{-i}, s_{i}\right)-v_{i}\left(s_{i}, s_{-i}\right) \leq 0$ we must have $\hat{s}_{-i} \geq s_{-i}$. By monotonicity, $v_{i}\left(s_{i}, \hat{s}_{-i}\right) \geq v_{i}\left(s_{i}, s_{-i}\right)=b$, hence $v_{-i}\left(s_{-i}, s_{i}\right) \geq b$. In either case there exists $\hat{s}_{-i}$ such that $v_{-i}\left(\hat{s}_{-i}, s_{i}\right) \geq b$ and the IVT now implies the existence of $s_{-i}^{\prime} \in\left[s_{-i}, \hat{s}_{-i}\right]$ for which $v_{-i}\left(s_{-i}^{\prime}, s_{i}\right)=b$. Thus $\varphi_{-i}\left(s_{i}, b\right)=s_{-i}^{\prime}$. The case of $v_{-i}\left(s_{-i}, s_{i}\right) \geq b$ is handled by first noting that this implies $s_{i} \leq \bar{s}_{i}$ and applying the analogous argument.

Finally, for the fourth claim, suppose for bidder 2 , say, that $\varphi_{2}(1, b)$ does not exist, i.e. there is no $s_{2}$ such that $v_{2}\left(1, s_{2}\right)=b$. Since $b \in B$ we know that $v_{2}(1,1) \geq b$, and hence by the IVT we must have $v_{2}\left(1, s_{2}\right)>b$ for every $s_{2}$. In particular, $v_{2}(1,0)>b$.

Observe that $v_{1}\left(\underline{s}_{1}, \underline{s}_{2}\right)=v_{2}\left(\underline{s}_{1}, \underline{s}_{2}\right)$, and hence by the single-crossing condition, $v_{1}\left(\underline{s}_{1}, 0\right) \geq v_{2}\left(\underline{s}_{1}, 0\right)$ since $0 \leq \underline{s}_{2}$. Applying the single-crossing condition again, we see that $v_{1}(1,0) \geq v_{2}(1,0)>b$. And since $v_{1}(0,0) \leq b$, the IVT yields a $s_{1}$ such that $v_{1}\left(s_{1}, 0\right)=b$, and $\varphi_{1}(0, b)=s_{1}$.

Define the following pair of functions. 


$$
\begin{aligned}
& m_{i}^{0}(b)= \begin{cases}\varphi_{i}\left(\bar{s}_{-i}, b\right) & \text { if it exists } \\
0 & \text { otherwise }\end{cases} \\
& M_{i}^{0}(b)= \begin{cases}\varphi_{i}\left(\underline{s}_{-i}, b\right) & \text { if it exists } \\
1 & \text { otherwise }\end{cases}
\end{aligned}
$$

It follows from parts 3 and 4 of Lemma 3.3 that for each $b \in B$ and $i=1,2$, $\varphi_{i}\left(m_{-i}^{0}(b), b\right)$ and $\varphi_{i}\left(M_{-i}^{0}(b), b\right)$ exist. We can thus inductively define

$$
\begin{aligned}
M_{i}^{k}(b) & =\varphi_{i}\left(m_{-i}^{k-1}(b), b\right) & k & =1,2, \ldots \\
m_{i}^{k}(b) & =\varphi_{i}\left(M_{-i}^{k-1}(b), b\right) & k & =1,2, \ldots
\end{aligned}
$$

which by Lemma 3.3 yield continuous functions of $b$ for each $k$.

Lemma 3.4. For all $k \geq 1$ and for all $b \in B$,

$$
\begin{aligned}
& \text { 1. } s_{i} \geq(\text { resp. }>) M_{i}^{k}(b) \Longrightarrow v_{i}\left(s_{i}, s_{-i}\right) \geq(\text { resp. }>) b \forall s_{-i} \geq m_{-i}^{k-1}(b) . \\
& \text { 2. } s_{i} \leq(\text { resp. }<) m_{i}^{k}(b) \Longrightarrow v_{i}\left(s_{i}, s_{-i}\right) \leq(\text { resp. }<) b \forall s_{-i} \leq M_{-i}^{k-1}(b) .
\end{aligned}
$$

Proof. We prove the first claim. The second is shown by the symmetric argument. If $s_{i}>M_{i}^{k}(b)$ and $s_{-i} \geq m_{-i}^{k-1}(b)$, then by the monotonicity of $v_{i}$, $v_{i}\left(s_{i}, s_{-i}\right) \geq v_{i}\left(s_{i}, m_{-i}^{k-1}(b)\right)>v_{i}\left(M_{i}^{k}(b), m_{-i}^{k-1}(b)\right)$ and the latter is equal to $b$ by definition.

We wish first to show that for each $b=b_{i}\left(s_{i}\right) \in B$, the sequences $M_{i}^{k}(b)$ and $m_{i}^{k}(b)$ for $i=1,2$, converge. By definition, $M_{-i}^{0}(b) \leq \bar{s}_{-i}$ and $m_{-i}^{0}(b) \geq \underline{s}_{-i}$. Hence by the monotonicity of $\varphi_{i}$ (part 2 of Lemma 3.3),

$$
M_{i}^{1}(b)=\varphi_{i}\left(m_{-i}^{0}(b), b\right) \leq M_{i}^{0}(b)
$$

because the latter is either $\bar{s}_{i}$ or $\varphi_{i}\left(\underline{s}_{-i}, b\right)$. Similarly $m_{i}^{1}(b) \geq m_{i}^{0}(b)$.

Now by the monotonicity of $\varphi_{i}$, we can inductively conclude that $m_{i}^{k}(b) \geq$ $m_{i}^{k-1}(b)$ and $M_{i}^{k}(b) \leq M_{i}^{k-1}(b)$ for all $k \geq 1$. Furthermore, since the range of $\varphi_{i}$ is $S_{i}$, these sequences are bounded. Hence for $i=1,2$, there are types $m_{i}^{*}(b)$ and $M_{i}^{*}(b)$ such that $m_{i}^{k}(b) \rightarrow m_{i}^{*}(b)$ and $M_{i}^{k}(b) \rightarrow M_{i}^{*}(b)$. 
Finally, since $v_{i}\left(m_{i}^{k}(b), M_{-i}^{k-1}(b)\right)=v_{i}\left(M_{i}^{k}(b), m_{-i}^{k-1}(b)\right)=b$, for all $k \geq 1$, the continuity of the valuation functions implies that for each $i$,

$$
v_{i}\left(m_{i}^{*}(b), M_{-i}^{*}(b)\right)=v_{i}\left(M_{i}^{*}(b), m_{-i}^{*}(b)\right)=b
$$

which in turn implies $m_{i}^{*}(b)=M_{i}^{*}(b)=s_{i}$.

To summarize the argument to this point, for each $s_{i} \in S_{i}$,

$$
\begin{gathered}
M_{i}^{k}\left(b_{i}\left(s_{i}\right)\right) \downarrow s_{i} \\
m_{i}^{k}\left(b_{i}\left(s_{i}\right)\right) \uparrow s_{i}
\end{gathered}
$$

We now describe an elimination sequence which yields an efficient dominance solution. As a first step, we eliminate any strategy $\rho$ such that for some $s_{i}<\bar{s}_{i}, \rho\left(s_{i}\right)>\bar{s}_{i}$ or for some $s_{i}>s_{i}, \rho\left(s_{i}\right)<s_{i}$. This can be done in the former case because a report $r_{i}>\bar{s}_{i}$ will win the auction against any report of the opponent, in particular, against a report $r_{-i}=\bar{s}_{-i}$. In this case, the payment would be $b_{i}\left(\bar{s}_{i}\right)=v_{i}\left(\bar{s}_{i}, \bar{s}_{-i}\right)>v_{i}\left(s_{i}, \hat{s}_{-i}\right)$ for any type $\hat{s}_{-i}$ of the opponent. The alternative report $\bar{s}_{i}$ for type $s_{i}$ would affect the allocation only in this case, and in this case would win with a lower probability. Since the net payoff from winning in this case was negative, this is a strict improvement. To summarize, for every type of the opponent, and for every report of the opponent, the report $\overline{s_{i}}$ does no worse than $r_{i}$, and does strictly better whenever the opponent reports $\bar{s}_{-i}$. A similar argument shows that a report of $r_{i}<s_{i}$ is dominated for a type $s_{i}>\underline{s}_{i}$ by the report $\underline{s}_{i}$.

Next, for each $k=1,2, \ldots$, define the following subsets of $S_{i} \times S_{i}$ :

$$
\begin{aligned}
D_{i}^{k} & =\left\{\left(s_{i}, r_{i}\right) \in[0,1] \times S_{i}: s_{i}>M_{i}^{k}\left(b\left(r_{i}\right)\right)\right\} \\
C_{i}^{k} & =\left\{\left(s_{i}, r_{i}\right) \in[0,1] \times S_{i}: s_{i}<m_{i}^{k}\left(b\left(r_{i}\right)\right)\right\}
\end{aligned}
$$

We observe that any such pair of sets describes a set of admissible bidding strategies for bidder $i$, namely, the set of bidding strategies $\rho(\cdot)$ such that for each $s_{i} \in S_{i}$,

$$
\left(s_{i}, \rho\left(s_{i}\right)\right) \notin C_{i}^{k} \cup D_{i}^{k}
$$

Let $\beta_{i}^{k}$ denote the set bidding strategies represented in this sense by the sets $C_{i}^{k}$ and $D_{i}^{k}$, and set $\beta^{-1}=R$.

Lemma 3.5. For each $k \geq 0$, every strategy not in $\beta_{i}^{k}$, is dominated within $\beta^{k-1}$, by a strategy in $\beta_{i}^{k}$. 
Proof. Consider any $\left(s_{i}, r_{i}\right) \in D_{i}^{k}$. Define

$$
Z=\left\{b_{i}\left(\hat{r}_{i}\right)>b_{i}\left(r_{i}\right): s_{i} \leq M_{i}^{k}\left(b_{i}\left(\hat{r}_{i}\right)\right)\right\}
$$

By the continuity of $b_{i}$ and $M_{i}^{k}, Z$ is closed. Suppose $Z \neq \emptyset$. Then the value $b_{i}\left(r_{i}^{*}\right)=\min Z$ is well-defined, and by continuity $s_{i}=M_{i}^{k}\left(b_{i}\left(r_{i}^{*}\right)\right) \geq m_{i}^{k}\left(b_{i}\left(r_{i}^{*}\right)\right)$ and thus $\left(s_{i}, r_{i}^{*}\right) \notin C_{i}^{k} \cup D_{i}^{k}$. We claim that reporting $r_{i}^{*}$ ex post dominates reporting $r_{i}$ for $s_{i}$.

To prove this, note that the report of $r_{i}^{*}$ changes the allocation relative to that which would obtain under $r_{i}$ if and only if bidder $-i$ reports some $r_{-i}$ where

$$
b_{-i}\left(r_{-i}\right) \in\left[b_{i}\left(r_{i}\right), b_{i}\left(r_{i}^{*}\right)\right]
$$

Note first that in all such cases, by reporting $r_{i}^{*} i$ wins the auction and pays $b_{-i}\left(r_{-i}\right)$ rather than losing the auction and paying nothing, as he would if he were to report $r_{i}$. And second, observe that given the strategies $\beta_{-i}^{k-1}$ for $-i$ that remain, a type $s_{-i}$ will report $r_{-i}$ only if $s_{-i} \geq m_{-i}^{k-1}\left(b_{-i}\left(r_{-i}\right)\right)$.

Consider any such $b_{-i}\left(r_{-i}\right)$. Suppose $s_{i}<M_{i}^{k}\left(b_{-i}\left(r_{-i}\right)\right)$. Then since $b_{-i}\left(r_{-i}\right) \in\left[b_{i}\left(r_{i}\right), b_{i}\left(r_{i}^{*}\right)\right]$, there is some type $\hat{s}_{i} \in\left[r_{i}, r_{i}^{*}\right]$ such that $b_{-i}\left(r_{-i}\right)=$ $b_{i}\left(\hat{s}_{i}\right)$ and thus

$$
s_{i}<M_{i}^{k}\left(b_{i}\left(\hat{s}_{i}\right)\right) .
$$

Now if $\hat{s}_{i}=r_{i}$, then $b_{i}\left(r_{i}\right)=b_{i}\left(\hat{s}_{i}\right)=b_{-i}\left(r_{-i}\right)$ and thus $M_{i}^{k}\left(b_{i}\left(r_{i}\right)\right)=M_{i}^{k}\left(b_{-i}\left(r_{-i}\right)\right)>$ $s_{i}$, which is a contradiction since $\left(s_{i}, r_{i}\right) \in D_{i}^{k}$. So $\hat{s}_{i} \in\left(r_{i}, r_{i}^{*}\right]$ and thus $b_{i}\left(\hat{s}_{i}\right) \in$ $\left(b_{i}\left(r_{i}\right), b_{i}\left(r_{i}^{*}\right)\right]$. Now by our supposition (2) and the definition of $r_{i}^{*}$ we must have $b_{i}\left(\hat{s}_{i}\right) \leq b_{i}\left(r_{i}^{*}\right)$, so in fact $b_{i}\left(\hat{s}_{i}\right)=b_{i}\left(r_{i}^{*}\right)$. But this implies $s_{i}=M_{i}^{k}\left(b_{i}\left(\hat{s}_{i}\right)\right)$, which contradicts (2). We thus conclude that our original supposition was false and $s_{i} \geq M_{i}^{k}\left(b_{-i}\left(r_{-i}\right)\right)$ for all $b_{-i}\left(r_{-i}\right) \in\left[b_{i}\left(r_{i}\right), b_{i}\left(r_{i}^{*}\right)\right]$.

By Lemma 3.4, it follows that for all $b_{-i}\left(r_{-i}\right) \in\left[b_{i}\left(r_{i}\right), b_{i}\left(r_{i}^{*}\right)\right], v_{i}\left(s_{i}, s_{-i}\right) \geq$ $b_{-i}\left(r_{-i}\right)$ for every $s_{-i} \geq m_{-i}^{k-1}\left(b_{-i}\left(r_{-i}\right)\right)$. Furthermore, since $s_{i}>M_{i}^{k}\left(b_{i}\left(r_{i}\right)\right)$, Lemma 3.4 implies that the inequality is strict for $b_{-i}\left(r_{-i}\right)=b_{i}\left(r_{i}\right)$. We have just shown that for all reports of $-i$ such that reporting $r_{i}^{*}$ wins the object where reporting $r_{i}$ would not, and for all types of $-i$ that could make such reports in $\beta_{-i}^{k-1}$, the value of the object exceeds its price, strictly in at least one case.. Thus, the report $r_{i}^{*}$ ex post dominates $r_{i}$ for $s_{i}$.

Now suppose $Z=\emptyset$. Then applying the same argument as above, $s_{i}>$ $M_{i}^{k}(b)$ for all $b \in\left[b_{i}\left(r_{i}\right), \bar{b}\right]$, implying that $v_{i}\left(s_{i}, \hat{s}_{-i}\right)>b$ for all such $b$ for every possible $\hat{s}_{-i}$ for which there remains a report $r_{-i}$ such that $b=b_{-i}\left(r_{-i}\right)$. Since 
truthful reporting is never eliminated, one such possibility is $\bar{s}_{-i}$, reporting $\bar{b}$. In this case $v_{i}\left(s_{i}, \bar{s}_{-i}\right)>\bar{b}=v_{i}\left(\bar{s}_{i}, \bar{s}_{-i}\right)$ and so monotonicity implies $s_{i}>\bar{s}_{i}$. In this case, $r_{i}$ is dominated for $s_{i}$ by the truthful report $s_{i}$. This report changes the allocation only in the above mentioned cases, where it ensures that $i$ wins the auction an pays $b_{-i}\left(r_{-i}\right)$, a price strictly less than his valuation.

In both cases, the dominating reports $r_{i}^{*}$ satisfy $\left(s_{i}, r_{i}^{*}\right) \notin D_{i}^{k} \cup C_{i}^{k}$. By a similar argument, it can be shown that for any $\left(s_{i}, r_{i}\right)$ in $C_{i}^{k}$, the report $r_{i}$ is dominated for $s_{i}$ by an alternative report $r_{i}^{*}$ for which $\left(s_{i}, r_{i}^{*}\right) \notin D_{i}^{k} \cup C_{i}^{k}$.

We have thus shown that for any type $s_{i} \in S_{i}$, if $\left(s_{i}, r_{i}\right) \in C_{i}^{k} \cup D_{i}^{k}$, then $r_{i}$ is ex post dominated for $s_{i}$ by an alternative report that is not in $C_{i}^{k} \cup D_{i}^{k}$. It follows that all bidding strategies other than those in $\beta_{i}^{k}$ are dominated by a strategy in $\beta_{i}^{k}$ and thus can be eliminated.

We can now conclude the proof of the existence of an efficient dominance solution of the generalized VCG mechanism. Let $\beta$ be the set of strategy profiles remaining after the elimination sequence $\beta^{k}$. A reporting strategy $\rho_{i}$ remains in $\beta_{i}$ for bidder $i$ iff

$$
\rho_{i}\left(s_{i}\right) \in \begin{cases}\left(\bar{s}_{i}, 1\right] & \text { for } s_{i}>\bar{s}_{i} \\ {\left[\bar{s}_{i}, 1\right]} & \text { for } s_{i}=\bar{s}_{i} \\ \left\{s_{i}\right\} & \text { for } s_{i} \in S_{i} \\ {\left[0, s_{i}\right]} & \text { for } s_{i}=\underline{s}_{i} \\ {\left[0, s_{i}\right)} & \text { for } s_{i}<\underline{s}_{i}\end{cases}
$$

Thus, for any profile of strategies within $\beta$, the allocation is identical, except possibly in the event that $s=\bar{s}$ or $s=\underline{s}$. However, in either of these two cases, the two bidders have the same valuation for the object, and for any strategy profile in $\beta$, the winner will pay that valuation. Thus, no strategies in $\beta$ are dominated within $\beta$. Note also that $\beta$ is an ex post equilibrium. Moreover, the allocation is ex post efficient. Finally, this elimination sequence is vigilant because every dominated strategy is eliminated at every stage.

We now establish that every dominance solution is an efficient ex post equilibrium. We will show that every vigilant dominance solution is a subset of $\beta$. Let $\hat{\beta}^{k}$ be an vigilant elimination sequence. Then there exists $K$ such that any strategy that is dominated in $K$ consecutive stages must be eliminated. We define

$$
\hat{\beta}_{i}^{k}\left(s_{i}\right)=\left\{\rho_{i}\left(s_{i}\right): \rho_{i} \in \hat{\beta}_{i}^{k}\right\} .
$$

We will make use of the following lemma: 
Lemma 3.6. For every $s_{i} \in S_{i}$

1. If $b=b_{-i}\left(s_{-i}\right)>b_{i}\left(s_{i}\right)$, then $v_{i}\left(s_{i}, s_{-i}\right)<b$.

2. If $b=b_{-i}\left(s_{-i}\right)<b_{i}\left(s_{i}\right)$, then $v_{i}\left(s_{i}, s_{-i}\right)>b$.

Proof. Consider the first claim. There is a $\hat{s}_{i}>s_{i}$ such that $v_{i}\left(\hat{s}_{i}, s_{-i}\right)=$ $b_{-i}\left(s_{-i}\right)$. By monotonicity, $v_{i}\left(s_{i}, s_{-i}\right)<b_{-i}\left(s_{-i}\right)=b$. The second claim follows from the symmetric argument.

The first step is to show by induction that no elimination sequence can eliminate a strategy $\rho_{i}$ specifying $\rho_{i}\left(s_{i}\right)=s_{i}$ for all $s_{i} \in S_{i}, \rho_{i}\left(s_{i}\right)>\bar{s}_{i}$ for all $s_{i}>\bar{s}_{i}$ and $\rho_{i}\left(s_{i}\right)<\underline{s}_{i}$ for all $s_{i}<\underline{s}_{i}$. We will call any such strategy a "truthtelling" strategy.

Obviously all truthtelling strategies belong to $\hat{\beta}^{0}$. Now consider any stage of elimination $k$ such that for each $i$ all truthtelling strategies remain in $\beta^{k-1}$. First consider any untruthful report $r_{i}$ for type $s_{i} \in\left(s_{i}, \overline{s_{i}}\right)$ of bidder $i$. If $r_{i}>s_{i}$, then $b_{i}\left(r_{i}\right)>b_{i}\left(s_{i}\right)$ and whenever $-i$ uses a truthtelling strategy $\rho_{-i}$, and his type $s_{-i}$ satisfies $b_{-i}\left(s_{-i}\right) \in\left(b_{i}\left(s_{i}\right), b_{i}\left(r_{i}\right)\right)$, bidder $i$ will win the auction and pay $b_{-i}\left(s_{-i}\right)$. By Lemma 3.6, $b_{-i}\left(s_{-i}\right)>v_{i}\left(s_{i}, s_{-i}\right)$. Since $i$ would have lost the auction in these cases with report $s_{i}$, truthtelling does strictly better. On the other hand, if $r_{i}<s_{i}$, then for any $b_{-i}\left(r_{-i}\right) \in\left(b_{i}\left(r_{i}\right), b_{i}\left(s_{i}\right)\right)$, bidder $i$ would lose the auction with $r_{i}$ whereas he would have won with $s_{i}$ and payed $b_{-i}\left(s_{-i}\right)$. In these cases, given truthelling by $-i$, $i$ 's payoff from winning would be strictly positive by Lemma 3.6, so again $s_{i}$ strictly prefers to tell the truth. We have shown that there is at least one possible case in $\hat{\beta}^{k-1}$ namely truthtelling by the opponent, such that $s_{i} \in\left(s_{i}, \bar{s}_{i}\right)$ strictly prefers to tell the truth. Thus, truthtelling by such types cannot be dominated. A slight modification of this argument delivers the same conclusion for types $s_{i}$ and $\bar{s}_{i}$. (For example, a report greater than $\overline{s_{i}}$ cannot dominate $\overline{s_{i}}$ because either report always leads to the same outcome.)

Next, given that truthelling is an element of $\hat{\beta}^{k-1}$, reports $r_{i}>\overline{s_{i}}$ for types $s_{i}>\bar{s}_{i}$ and $r_{i}<s_{i}$ for types $s_{i}<s_{i}$ cannot be eliminated. To see this note that if, say, type $s_{i}>\overline{s_{i}}$ reports $\hat{r}_{i} \leq \overline{s_{i}}$ rather than $r_{i}>\overline{s_{i}}$ (other alternatives can never change the allocation), then in the event that the opponent's type is $\bar{s}_{-i}$ (which is 1 in this case) and reports truthfully, $i$ will lose the auction with $\hat{r}_{i}$ rather than win with $r_{i}$ and pay $\bar{b}=v_{i}\left(\bar{s}_{i}, \bar{s}_{-i}\right)<v_{i}\left(s_{i}, \bar{s}_{-i}\right)$. Since there is at least one case in which $r_{i}$ leads to a strictly lower payoff than $r_{i}$, we conclude that $\hat{r}_{i}$ cannot dominate $r_{i}$. A similar argument shows that no reports below $s_{i}$ can be dominated for a type $s_{i}<s_{i}$. 
We have shown that no truthtelling strategy can be eliminated in any round. Thus, every dominance solution $\hat{\beta}$ is non-empty and includes all truthtelling strategies. We will now show that $\hat{\beta} \subset \beta$. To do so, we will show that there is no strategy $\rho_{i}$ in $\hat{\beta}$ satisfying any of the following:

1. $\rho_{i}\left(s_{i}\right)=\hat{s}_{i} \in S_{i}$ for some $s_{i} \neq \hat{s}_{i}$

2. $\rho_{i}\left(s_{i}\right)>\overline{s_{i}}$ for some $s_{i}<\overline{s_{i}}$

3. $\rho_{i}\left(s_{i}\right)<\underline{s}_{i}$ for some $s_{i}>\underline{s}_{i}$

To show that 2 cannot hold for any $\rho_{i}$ in $\hat{\beta}$ observe that the domination argument used in the first round of elimination leading to $\beta$ in the proof of Lemma 3.5 applies in any round in which all truthtelling strategies survive. Thus, if $s_{i}<\overline{s_{i}}$ and $r_{i}>\bar{s}_{i}$, then $r_{i}$ is dominated for $s_{i}$ by $\overline{s_{i}}$ in every round.

Suppose $r_{i}$ is not eliminated in any round for type $s_{i}$. Then by vigilance, it must be the case that in some round $k \leq K$, reporting $\overline{s_{i}}$ is eliminated for type $s_{i}$. Let $k$ be the first round in which reporting $\overline{s_{i}}$ is eliminated for type $s_{i}$. Then there is some report $\hat{r}_{i}$, which survives for type $s_{i}$ in round $k$ and dominates reporting $\bar{s}_{i}$ within $\hat{\beta}^{k-1}$. This means that for every $b \in\left[b_{i}\left(\hat{r}_{i}\right), b_{i}\left(\bar{s}_{i}\right)\right]$, and for every $s_{-i}$ such that $b \in b_{-i}\left(\hat{\beta}_{-i}^{k-1}\left(s_{-i}\right)\right)$, we have $v_{i}\left(s_{i}, s_{-i}\right) \leq b$. This implies, by lemma 3.6 and the fact that all truthtelling strategies remain, that $\hat{r}_{i} \geq s_{i}$. Applying lemma 3.6 again, we conclude that for every $b$ in the interior of this interval, $v_{i}\left(s_{i}, s_{-i}\right)<b$ for $b_{-i}\left(s_{-i}\right)=b$. Since the report of $\hat{r}_{i}$ changes the allocation relative to $r_{i}$ only when $b_{-i}\left(r_{-i}\right) \in\left[b_{i}\left(\hat{r}_{i}\right), b_{i}\left(\bar{s}_{i}\right)\right]$ and in all such cases the payoff to bidder $i$ is non-positive and in some cases strictly negative, $\hat{r}_{i}$ dominates $r_{i}$ as well. We can iterate this argument to conclude that for every $k, r_{i}$ is dominated within $\hat{\beta}^{k-1}$ by an element of $\hat{\beta}^{k}$. Therefore, by vigilance, $r_{i} \notin \hat{\beta}_{i}\left(s_{i}\right)$. An analogous argument shows that 3 cannot hold.

Finally, we show that 1 cannot be true of any $\rho_{i}$ in $\hat{\beta}$. We will do this by demonstrating that for every $k$, every $\left(s_{i}, r_{i}\right) \in D_{i}^{k} \cup C_{i}^{k}$ is eventually eliminated. The argument is by induction. Let $\left(s_{i}, r_{i}\right) \in D_{i}^{1} \cup C_{i}^{1}$. Then $r_{i}$ is dominated within $\hat{\beta}^{0}$ for $s_{i}$ by some $r_{i}^{0} \neq r_{i}$. By the same argument as above, $r_{i}^{0}$ dominates $r_{i}$ for $s_{i}$ within $\hat{\beta}^{k}$ for every $k$.

Suppose $r_{i}^{0} \in \hat{\beta}_{i}^{K}\left(s_{i}\right)$. Then by vigilance, since $r_{i}$ could be eliminated in $K$ consecutive stages, $r_{i}$ must be eliminated for $s_{i}$ in $\hat{\beta}_{i}^{K}$. On the other hand, if $r_{i}^{0} \notin \hat{\beta}_{i}^{K}\left(s_{i}\right)$, then there is a sequence of reports $r_{i}^{0}, r_{i}^{1}, \ldots, r_{i}^{T}$, such that $r_{i}^{T} \in \hat{\beta}_{i}^{K}\left(s_{i}\right)$, and for each $t=0, \ldots, T-1$, there is a stage $k(t)$ such that $r_{i}^{t}$ was 
eliminated in stage $k(t)$ because it was dominated within $\hat{\beta}^{k(t)}$ by $r_{i}^{t+1} \in \hat{\beta}^{k(t)+1}$. We can now argue just as above that $r_{i}^{0}$ is dominated successively by each $r_{i}^{t}$, and hence that $r_{i}^{0}$ could have been eliminated for $K$ consecutive stages. By vigilance, $r_{i}^{0} \notin \hat{\beta}_{i}^{K}\left(s_{i}\right)$. Thus all reporting strategies in $\beta_{i}^{0}$ will be eliminated by stage $K$.

Now suppose that for a given $\bar{k}$, all strategies in $\beta^{0} \backslash \beta^{\bar{k}}$ are eliminated by round $\bar{k} K$, and let $\left(s_{i}, r_{i}\right) \in C_{i}^{\bar{k}+1} \cup D_{i}^{\bar{k}+1}$. By lemma $3.5, r_{i}$ is dominated within $\hat{\beta}^{\bar{k} K}$ for $s_{i}$ by some $r_{i}^{0}$. If $r_{i}^{0} \in \hat{\beta}^{(\bar{k}+1) K}$, then $r_{i}$ could be eliminated in each of the $K$ intervening stages and by vigilance would be eliminated in stage $(\bar{k}+1) K$.

If $r_{i}^{0} \notin \hat{\beta}^{(\vec{k}+1) K}$, then there exists a sequence of reports $r_{i}^{0}, r_{i}^{1}, \ldots, r_{i}^{T}$, corresponding to stages $k(t)$ for $t=0, \ldots, T-1$ with $r_{i}^{T} \in \hat{\beta}^{(\bar{k}+1) K}$ and with each $r_{i}^{t}$ eliminated in stage $k(t)$, dominated within $\hat{\beta}^{k(t)}$ by $r_{i}^{t+1} \in \hat{\beta}^{k(t)+1}$. Then by the same transitivity argument used previously, $r_{i}^{0}$ is dominated successively by each $r_{i}^{t}$ and hence $r_{i}$ could be eliminated in each stage from $\bar{k} K+1$ to $(\bar{k}+1) K$ and hence by vigilance must be eliminated in the latter stage.

Since $\left(s_{i}, r_{i}\right)$ was arbitrary, we have shown that all strategies within $\beta^{\bar{k}} \backslash$ $\beta^{\bar{k}+1}$ must be eliminated by stage $(\bar{k}+1) K$, and this concludes the inductive step and the proof.

\section{MULTI-UNIT AUCTIONS WITH TWO BIDDERS}

The essential equivalence between implementability in ex post equilibrium and ex post dominance implementability of efficient allocation generalizes straightforwardly to multi-unit auctions with two bidders. Suppose there are $L \geq 1$ identical objects. For any $i \in \mathscr{I}$ and $1 \leq l \leq L$, let $v_{i}^{l}: T \rightarrow \mathbf{R}_{+}$ be bidder $i$ 's marginal valuation of the $l$ th object he wins. We assume nonincreasing marginal valuations: $\left[1 \leq l<l^{\prime} \leq L\right] \Longrightarrow\left[v_{i}^{l} \geq v_{i}^{l^{\prime}}\right]$. We maintain the assumption that $v_{i}^{l}$ is continuous, strictly increasing, and satisfies the following single-crossing condition:

Assumption 2 (Single-Crossing Property). For any $1 \leq l \leq L$, for any $i \neq j$ and $\hat{s}_{j}$, the difference

$$
g\left(s_{i}\right)=v_{i}^{l}\left(s_{i}, \hat{s}_{j}\right)-v_{j}^{L+1-l}\left(s_{i}, \hat{s}_{j}\right)
$$

crosses zero at most once, and from below. 
Theorem 4.1. When there are two bidders and $L \geq 1$ identical objects, the efficient allocation is ex post dominance implementable.

Proof: Label the $L$ objects from 1 to $L$. Consider the following indirect version of the generalized VCG mechanism. We imagine $L$ simultaneous auctions. For each auction $l$ there will correspond sets $S_{i}^{l}$ and $B^{l}=\left[\underline{b}^{l}, \bar{b}^{l}\right]$ and mappings $b_{i}^{l}(\cdot)$ constructed just as in the single-unit case, now using the valuation functions $v_{1}^{l}(\cdot)$ and $v_{2}^{L-l+1}(\cdot)$. For notational convenience below, we will modify the definition of $b_{i}^{l}$ outside of $S_{i}^{l}$ by defining

$$
\begin{aligned}
& \bar{b}=1+\max _{i, l, s} v_{i}^{l}(s) \\
& \underline{b}=-1+\min _{i, l, s} v_{i}(s)
\end{aligned}
$$

and setting $b_{i}^{l}(s)=\bar{b}$ for $s_{i}>\bar{s}_{i}^{l}$ and $b_{i}^{l}(s)=\underline{b}$ for $s_{i}<s_{i}^{l}$.

Each bidder simultaneously submits $L$ reports $r_{i}^{1}, \ldots, r_{i}^{L}$. with the restriction that $b_{1}^{1}\left(r_{1}^{1}\right) \leq, \ldots \leq b_{1}^{L}\left(r_{1}^{L}\right)$ and $b_{2}^{1}\left(r_{2}^{1}\right) \geq \ldots \geq b_{2}^{L}\left(r_{2}^{L}\right)$. Let $\Delta_{i}$ denote the set of reporting strategies for $i$ meeting the corresponding restriction. The idea is that for some $\hat{l}$, bidder 1 should win objects 1 through $\hat{l}$, and bidder 2 objects $\hat{l}+1$ through $L$. Thus object $l$ is awarded to the bidder with the greater imputed valuation according to the reports $r^{l}$ using the marginal valuation functions $v_{1}^{l}, v_{2}^{L-l+1}$. Ties will always be broken in favor of bidder $1^{10}$ The payment of the winner of object $l$ is calculated in the same way as the single unit VCG auction, using these reports and marginal valuation functions.

For each $l$ and for each $k=0,1 \ldots$, we define the functions $m_{i, l}^{k}(\cdot)$ and $M_{i, l}^{k}(\cdot)$ on the sets $B^{l}$ as before. By the same argument as in the single object case, these functions converge pointwise to e.g. $m_{1, l}^{*}(b)$ where $b_{1}^{l}\left(m_{1, l}^{*}(b)\right)=b$. Moreover,

Lemma 4.2. For every $l<L$, and for $k \geq 0$

1. $\underline{s}_{1}^{l} \leq \underline{s}_{1}^{l+1}, \bar{s}_{1}^{l} \leq \bar{s}_{1}^{l+1}, \underline{s}_{2}^{l} \geq \underline{s}_{2}^{l+1}$, and $\bar{s}_{2}^{l} \geq \bar{s}_{2}^{l+1}$.

2. $m_{1, l}^{k}(b) \leq m_{1, l+1}^{k}(b), M_{1, l}^{k}(b) \leq M_{1, l+1}^{k}(b), m_{2, l}^{k}(b) \geq m_{2, l+1}^{k}(b), M_{2, l}^{k}(b) \geq$ $M_{2, l+1}^{k}(b)$.

${ }^{10}$ This simplifies the notation in the proof. In general, any tie-breaking rule of the following form will suffice. Prior to receiving reports, the auctioneer chooses an integer $t \in\{0, \ldots, L\}$. It can be kept secret or revealed to the bidders. If there is a tie in auction $l$, then object $l$ is awarded to bidder 1 if and only if $l \leq t$. 
Proof. The first follows from the assumption of non-increasing marginal valuations. Non-increasing marginal valuations also implies that the second claim holds for $k=0$. Finally, the non-increasing marginal valuations and the monotonicity of $\varphi_{i}$ implies that the same inequalities are satisfied for every $k>0$.

The first step of elimination is to delete all reporting strategies $\rho_{i}$ such that for some $l$ and for some $s_{i} \in\left(\underline{s}_{i}^{l}, \bar{s}_{i}^{l}\right), \rho_{i}^{l}\left(s_{i}\right) \notin S_{i}^{l}$. For example, take $s_{1}<\bar{s}_{1}^{l}$ and suppose $\rho_{1}^{l}\left(s_{1}\right)>\bar{s}_{1}^{l}$ so that $b_{1}^{l}\left(\rho_{1}^{l}\left(s_{1}\right)\right)=\bar{b}$. And suppose $l$ is the smallest index for which these two inequalities are satisfied for $s_{1}$. We will show that $\rho_{1}\left(s_{1}\right)$ is dominated for $s_{1}$ by the following list of reports $\hat{r_{1}}$.

$$
{\hat{r_{1}}}^{\lambda}= \begin{cases}\max \left\{r \leq \rho_{1}^{\lambda}\left(s_{1}\right): b_{1}^{\lambda}(r) \leq \bar{b}^{l}\right\} & \lambda \geq l \\ \rho_{1}^{\lambda}\left(s_{1}\right) & \lambda<l\end{cases}
$$

By construction, $\hat{r_{1}} \in \Delta_{1}$. To show that it dominates $\rho_{1}\left(s_{1}\right)$, note that $\hat{r_{1}}$ yields a different allocation than $\rho_{1}\left(s_{1}\right)$ only in the event that bidder 2 submits a list of reports $r_{2}$ such that for some auction $\lambda$,

$$
b_{1}^{\lambda}\left(\hat{r}_{1}^{\lambda}\right) \leq b_{2}^{\lambda}\left(r_{2}^{\lambda}\right) \leq b_{1}^{\lambda}\left(\rho_{1}^{\lambda}\left(s_{1}\right)\right)
$$

with at least one of the inequalities strict. Notice that this can only be true for $\lambda \geq l$ since it is only in these auctions that bidder 1's report has changed. And in this case, since $\hat{r}_{1}^{\lambda} \neq \rho_{1}^{\lambda}\left(s_{1}\right)$ by definition we must have $b_{1}^{\lambda}\left(\hat{r}_{1}^{\lambda}\right)=\bar{b}^{l}$. Furthermore, for $\lambda<l, b_{1}^{\lambda}\left(\rho_{1}^{\lambda}\left(s_{1}\right)\right) \geq b_{1}^{l}\left(\rho_{1}^{l}\left(s_{1}\right)\right)=\bar{b}$, implying that bidder 1 will win the first $l-1$ objects for sure, and hence his marginal valuation for any additional object is no greater than $v_{1}^{l}$.

Now consider a $\lambda$ satisfying (3). For any $s_{2}$, we have

$$
v_{1}^{\lambda}(s) \leq v_{1}^{l}(s)<\bar{b}^{l},
$$

where the first inequality follows from the assumption of declining marginal valuations. To demonstrate the second inequality, observe that $\rho_{1}^{l}\left(s_{1}\right)>\bar{s}_{1}^{l}$ implies that $\bar{s}_{1}^{l}<1$ so that $\bar{s}_{2}^{l}=1$. Hence, $\bar{b}^{l}=v_{1}^{l}\left(\bar{s}_{1}, 1\right)>v_{1}^{l}(s)$ by monotonicity of $v_{1}^{l}$. Since $\bar{b}^{l} \leq b_{2}^{\lambda}\left(r_{2}^{\lambda}\right)$, and the latter is the price bidder 1 would have to pay for object $\lambda$, we have just shown that bidder 1's marginal valuation for any additional objects is strictly less than their price for any possible type of bidder 2 . Thus, by lowering his reports to $\hat{r_{1}}$, against any strategy of 1 for which the 
allocation is altered, 1 strictly reduces the probability of earning a negative payoff. Thus, $\hat{r}_{1}$ ex post dominates $\rho_{1}\left(s_{1}\right)$ for $s_{1} \cdot{ }^{11}$

By a similar argument we can eliminate any reporting strategy $\rho_{1}$ such that $\rho_{1}^{l}\left(s_{1}\right)<\underline{s}_{1}^{l}$ for some $l$ and $s_{1}>\underline{s}_{1}^{l}$. Such a reporting strategy will be dominated by one in which the report to the $l$ th auction is raised to $\underline{s}_{1}^{l}$, with reports to all auctions $\lambda<l$ correspondingly raised in order to satisfy feasibility. This change affects the allocation only when $b_{2}^{\lambda}\left(r_{2}^{\lambda}\right) \leq \underline{b}^{l}$ for some $\lambda \leq l$, in which case 1 wins the auction and pays no more than $\underline{b}^{l}$. In this case, we can argue analogously to the above that bidder 1's valuation always strictly exceeds this price and hence the change only increases the probability of a positive payoff. The symmetric pair of arguments applies to bidder 2 .

We construct the remainder of the elimination sequence as follows. For each $k=0,1, \ldots$, define the following nested sequence of strategy sets for each bidder $i$

$$
\begin{aligned}
& \beta_{i}^{k}=\left\{\rho_{i} \in \Delta_{i}: b_{i}^{l}\left(\rho_{i}^{l}\left(s_{i}\right)\right)=b \in B^{l} \Longrightarrow\right. \\
& \left.s_{i} \in\left[m_{i, l}^{k}(b), M_{i, l}^{k}(b)\right] \quad l=1, \ldots, L\right\}
\end{aligned}
$$

Let $\beta^{k}$ be the set of profiles $\beta_{1}^{k} \times \beta_{2}^{k}$, and set $\beta^{-1}=\Delta$.

Lemma 4.3. For each $k \geq 0$, every strategy not in $\beta_{i}^{k}$, is dominated within $\beta^{k-1}$, by a strategy in $\beta_{i}^{k}$.

Proof. Consider first a reporting strategy $\rho_{1}$ such that $\rho_{1}\left(s_{1}\right)=r_{1}$ for a type $s_{1}$ of bidder 1 . Suppose for some auction $\lambda$,

$$
b_{1}^{\lambda}\left(r_{1}^{\lambda}\right) \in B^{\lambda} \text { but } s_{1}<m_{1, \lambda}^{k}\left(b_{1}^{\lambda}\left(r_{1}^{\lambda}\right)\right) .
$$

And suppose $l$ is the highest index $\lambda$ for which this is true for $s_{1}, r_{1}$. Let

$$
Z=\left\{\hat{r}_{1}^{l} \in\left[\underline{s}_{1}^{l}, r_{1}^{l}\right]: s_{1} \geq m_{1, l}^{k}\left(b_{1}^{l}\left(r_{1}^{l}\right)\right)\right\}
$$

by the continuity of $b_{1}^{l}$ and $m_{1, l}, Z$ is closed.

Suppose $Z \neq \emptyset$. Then define $r_{1}^{*}=\max Z$, and consider the alternative list of reports $\hat{r}_{1}=\left(r_{1}^{1}, \ldots, r_{1}^{*}, \ldots, r_{1}^{L}\right)$. We claim first that $\hat{r}_{1} \in \Delta_{1}$.

11 The report list $\hat{r_{1}}$ may itself be dominated. However, it is straightforward to extend the arguments from the single-unit case to find an undominated strategy that dominates $\rho_{1}$ for type $s_{1}$. 
Suppose

$$
b_{1}^{l+1}\left(r_{1}^{l+1}\right)>b_{1}^{l}\left(r_{1}^{*}\right)
$$

Then

$$
b_{1}^{l+1}\left(r_{1}^{l+1}\right)>\underline{b} .
$$

Since $r_{1}$ was feasible,

$$
b_{1}^{l}\left(r_{1}^{l}\right) \geq b_{1}^{l+1}\left(r_{1}^{l+1}\right) .
$$

It follows that $b_{1}^{l+1}\left(r_{1}^{l+1}\right) \leq \bar{b}^{l+1}$ otherwise $b_{1}^{l+1}\left(r_{1}^{l+1}\right)=\bar{b}>\bar{b}^{l} \geq b_{1}^{l}\left(r_{1}^{l}\right)$ which contradicts (8). Combining this with (7), we have shown that $b_{1}^{l+1}\left(r_{1}^{l+1}\right) \in B^{l+1}$. Thus, $b_{1}^{l+1}\left(r_{1}^{l+1}\right)$ is well defined and

$$
s_{1} \geq m_{1, l+1}^{k}\left(b_{1}^{l+1}\left(r_{1}^{l+1}\right)\right) \geq m_{1, l}^{k}\left(b_{1}^{l+1}\left(r_{1}^{l+1}\right)\right)
$$

where we have the first inequality by the definition of $l$ and the second inequality is an application of lemma 4.2. But now (6), (8) and (9) contradict the definition of $r_{1}^{*}$. Thus $\hat{r}_{1}$ is feasible.

We now show that $\hat{r}_{1}$ dominates $r_{1}$ for $s_{1}$. First note that $\hat{r}_{1}$ changes the allocation relative to $r_{1}$ only if bidder 2's report $r_{2}$ satisfies

$$
b_{2}^{l}\left(r_{2}^{l}\right) \in\left(b_{1}^{l}\left(\hat{r}_{1}^{l}\right), b_{1}^{l}\left(r_{1}^{l}\right)\right]
$$

By construction of $\hat{r_{1}}$, we have $s_{1}<m_{1, l}^{k}\left(b_{2}\left(r_{2}\right)\right)$ for every such $r_{2}$. And in these cases, bidder 1 will lose the auction for object $l$ rather than winning and paying price $b_{2}^{l}\left(r_{2}^{l}\right)$.

Since for any $\lambda \leq l$

$$
b_{1}^{\lambda}\left(r_{1}^{\lambda}\right) \geq b_{1}^{l}\left(r_{1}^{l}\right) \geq b_{2}^{l}\left(r_{2}^{l}\right) \geq b_{2}^{\lambda}\left(r_{2}^{\lambda}\right)
$$

bidder 1 will win $^{12}$ all objects $1, \ldots, l-1$. Furthermore, for every $\lambda>l$,

$$
b_{1}^{\lambda}\left(r_{1}^{\lambda}\right) \leq b_{1}^{l}\left(\hat{r}_{1}^{l}\right)<b_{2}^{l}\left(r_{2}^{l}\right) \leq b_{2}^{\lambda}\left(r_{2}^{\lambda}\right)
$$

bidder 1 will lose all objects $l+1, \ldots L$. Thus, the change from $r_{1}$ to $\hat{r_{1}}$ affects only the allocation of object $l$ and bidder 1 's marginal valuation for object $l$ is given by $v_{1}^{l}(\cdot)$.

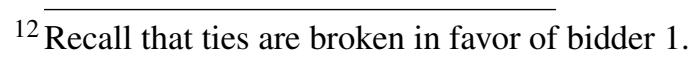


For any reporting strategy $\rho_{2}$ for bidder 2 in the set $\beta_{2}^{k-1}$, if $\rho_{2}^{l}\left(s_{2}\right)=r_{2}^{l}$, then bidder 2's type $s_{2}$ must be no greater than $M_{2, l}^{k-1}\left(b_{2}^{l}\left(r_{2}^{l}\right)\right)$. And since $s_{1}<m_{1, l}^{k}\left(b_{2}^{l}\left(r_{2}^{l}\right)\right)$, Lemma 3.4 implies that for every such $s_{2}$,

$$
v_{1}^{l}\left(s_{1}, s_{2}\right)<b_{2}^{l}\left(r_{2}^{l}\right) .
$$

Thus, for any $s_{2}$ that would report $r_{2}^{l}$, the marginal payoff to bidder 1 from winning object $l$ is strictly negative. Therefore in all cases in which $\hat{r_{1}}$ changes the allocation relative to $r_{1}$, it strictly increases the payoff of bidder 1 .

Now suppose $Z=\emptyset$. Then $s_{1}<m_{1, l}^{k}(b)$ for all $b \in\left[\underline{b}^{l}, b_{1}^{l}\left(r_{1}^{l}\right)\right]$. This implies in particular that $s_{1}<\underline{s}_{1}^{l}$ because if $s_{1} \geq \underline{s}_{1}^{l}$, then $s_{1} \geq m_{1, l}^{k}\left(\underline{b}^{l}\right)$. And by Lemma 4.2, $s_{1}<\underline{s}_{1}^{\lambda}$ for all $\lambda \geq l$. We can show by the same argument as above that for any report $r_{2}$ such that $b_{2}^{l}\left(r_{2}^{l}\right) \in\left[\underline{b}^{l}, b_{1}^{l}\left(r_{1}^{l}\right)\right]$, bidder 1 will win all objects $1, \ldots, l-1$, and for any type $s_{2}$ of bidder 2 such that the report $r_{2}$ is possible in $\beta_{2}^{k-1}$, bidder 1 's marginal valuation for the $l$ th object is strictly less than $b_{2}^{l}\left(r_{2}^{l}\right)$, the price.

Since for all $\lambda \geq l, m_{1, \lambda}^{k}(b) \geq m_{1, l}^{k}(b)$ and $b_{2}^{\lambda}\left(r_{2}^{\lambda}\right) \geq b_{2}^{l}\left(r_{2}^{l}\right)$, the payoff for all objects $\lambda>l$ is strictly negative as well. It follows that $r_{1}$ is dominated for $s_{1}$ by the report $\hat{r}_{1}$ defined as follows.

$$
{\hat{r_{1}}}^{\lambda}= \begin{cases}r_{1}^{\lambda} & \text { if } \lambda<l \\ s_{1} & \text { if } \lambda \geq l\end{cases}
$$

Obviously $\hat{r}_{1} \in \Delta_{1}$. And since $s_{1}<\underline{s}_{1}^{\lambda}$, and hence $b_{1}^{\lambda}\left(\hat{r}_{1}^{\lambda}\right)=\underline{b}$ for all $\lambda \geq l$, by reporting $\hat{r_{1}}$, bidder 1 is guaranteed to lose auctions $l, \ldots, L$, and avoid the strictly negative payoff. Thus $\hat{r_{1}}$ dominates $\rho_{1}\left(s_{1}\right)$ for type $s_{1}$.

To complete the domination argument, let $\left\{\lambda_{1}, \ldots, \lambda_{K}\right\}$ be the set of indices of auctions for which (5) holds. By definition, $\lambda_{K}=l$, and we have just shown that $\rho_{1}\left(s_{1}\right)$ is dominated for $s_{1}$ by a list of reports, call it $\tilde{r}(K)$ for which (5) is not satisfied for any $\lambda \geq \lambda_{K}$. By repitition of the domination argument above, $\tilde{r}(K)$ is dominated by a report list $\tilde{r}(K-1)$ for which (5) is not satisfied for any $\lambda \geq \lambda_{K-1}$. By induction, we arrive at a list of reports $\tilde{r}(1)$ for which (5) is not satisfied for any auction, and which dominates $\tilde{r}(2)$ and by the transitivity of the dominance relation, dominates $\rho_{1}\left(s_{1}\right)$.

Now consider the set of auction indices $\mu$ for which

$$
b_{1}^{\mu}\left(\tilde{r}^{\mu}(1)\right) \in B^{\mu} \text { but } s_{1}>M_{1, \mu}^{k}\left(b_{1}^{\mu}\left(\tilde{r}^{\mu}(1)\right)\right) .
$$


Let $l$ be the lowest index $\mu$ for which (10) is satisfied for $s_{1}, \tilde{r}(1)$. By an induction argument similar to the one in the previous paragraph, we can then show that $\tilde{r}(1)$, and by transitivity $\rho_{1}\left(s_{1}\right)$, is dominated by a report list, call it $\rho_{1}^{\prime}\left(s_{1}\right)$ for which neither (5) nor (10) are satisfied for any auction.

Since $s_{1}$ was arbitrary, we have shown that for any $\rho_{1}$ outside $\beta_{1}^{k}$, there is a $\rho_{1}^{\prime}$ in $\beta_{1}^{k}$ which dominates it within $\beta^{k-1}$.

We can now conclude the proof of theorem 4.1. Let $\beta$ be the set of strategy profiles that remain after the elimination sequence $\beta^{k}$. A reporting strategy $\rho_{i}$ remains in $\beta_{i}$ for bidder $i$ iff $\rho_{i} \in \Delta_{i}$ and, for all $l$,

$$
\rho_{i}^{l}\left(s_{i}\right) \in \begin{cases}\left(\bar{s}_{i}^{l}, 1\right] & \text { for } s_{i}>\bar{s}_{i}^{l} \\ {\left[\bar{s}_{i} l, 1\right]} & \text { for } s_{i}=\bar{s}_{i}^{l} \\ \left\{s_{i}\right\} & \text { for } s_{i} \in S_{i}^{l} \\ {\left[0, s_{i} l\right]} & \text { for } s_{i}=\underline{s}_{i}^{l} \\ {\left[0, s_{i}^{l}\right)} & \text { for } s_{i}<\underline{s}_{i}^{l}\end{cases}
$$

Clearly this is an efficient ex-post equilibrium. The proof that any vigilant elimination sequence leads to a subset of $\beta$ follows lines identical to the single-unit case and is omitted.

\section{AUCTIONS WITH $N \geq 3$ BIDDERS}

We have shown that when there are two bidders, the standard single crossing condition, essentially necessary for existence of an efficient ex post equilibrium, is sufficient for ex post dominance implementation. In this section, we show that with more than two bidders, conditions for ex post dominance implementability are generally strictly stronger than single crossing. To obtain easily interpretable necessary and sufficient conditions for ex post dominance implementation, we specialize in this section to symmetric linear valuation functions. Specifically, we assume the valuation functions take the following form.

$$
\forall i \in \mathscr{I}, v_{i}=a s_{i}+\sum_{j \neq i} s_{j}
$$

For this symmetric linear setting, there exists an efficient ex post equilibrium of the generalized VCG mechanism if and only if $a \geq 1$.Moreover, any direct revelation mechanism which has an efficient ex post equilibrium is a generalized VCG mechanism. Thus, to search for ex post dominance 
implementing direct mechanisms, it suffices to consider generalized VCG mechanisms.

The generalized VCG mechanism takes the following form. Each bidder $i$ reports his type (i.e. $A_{i}=T_{i}$ ). If $r$ is the profile of reports, each bidder's valuation is calculated assuming $r$ was truthful, i.e. $v_{i}=v_{i}(r)$. If $v_{i}>v_{j}$ for each $j \neq i$, then $i$ is awarded the object $\left(p_{i}(r)=1\right)$. In the event of ties, $p_{i}(r)$ can be chosen arbitrarily. In the symmetric linear setting with $a \geq 1$, the winner is the bidder reporting the highest type. The payment is determined as follows. Let bidder $i$ be the winner of the object, and let $\hat{r}_{i}=\min \left\{s_{i} \in S_{i} \mid\right.$ $\left.\forall j \in \mathscr{I}, v_{i}\left(s_{i}, r_{-i}\right) \geq v_{j}\left(s_{i}, r_{-i}\right)\right\}$. Then $t_{i}(r)=v_{i}\left(\hat{r}_{i}, r_{-i}\right)+f_{i}\left(r_{-i}\right)$ and $\forall j \neq i$, $t_{j}(r)=f_{j}\left(r_{-j}\right)$, where $f_{i}$ and the $f_{j}$ 's are arbitrary functions measurable only to $r_{-i}$ and the $r_{-j}$ 's, respectively.

The following proposition states that, in auctions with $n \geq 3$ bidders, the generalized VCG mechanism ex post dominance implements the efficient allocation only if a bidder's valuation is sufficiently sensitive to his own signal.

Proposition 5.1. In the symmetric linear setting with $n \geq 3$ bidders, the generalized VCG mechanism ex post dominance implements the efficient allocation if and only if $a>n-1$.

Proof. We first prove that, whenever $a>n-1$, truth-telling is a dominance solution of the generalized VCG mechanism with all the $f_{i}$ 's identically zero. Since truth-telling induces efficient allocation whenever $a>1$, this will finish the proof of the "if" part. ${ }^{13}$ We then prove that the generalized VCG mechanism, for any arbitrary $f_{i}$ 's, is not dominance solvable if $a \leq n-1$.

To begin with, we show that iteratively undominated reports must lie above some lower bound. Say that bidder $i$ is pivotal at report $r_{j}$ if $i$ has reported the highest type and the second highest report is $r_{j}$.

Suppose that type $s_{i}$ of bidder $i$ is pivotal at report $r_{j}$. Then the maximum report among the other bidders is $r_{j}$, and $i$ 's payment is at most $(a+n-1) r_{j}$. Bidder $i$ 's payoff conditional on being pivotal at $r_{j}$ is therefore at least $a s_{i}-$ $(a+n-1) r_{j}$. This payoff is zero for $r_{j}=\underline{r}\left(s_{i}\right)$ where

$$
\underline{r}\left(s_{i}\right)=\frac{a s_{i}}{a+n-1}
$$

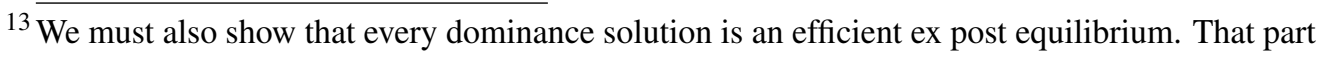
of the proof would be similar to the corresponding part of the proof of theorem 3.1. 
Note also that this minimum payoff is strictly positive for all reports less than $\underline{r}\left(s_{i}\right)$. It follows that any report below the lower bound $\underline{r}\left(s_{i}\right)$ is dominated by $\underline{r}\left(s_{i}\right)$. Note that $\underline{r}(0)=0$.

Now, assuming that each bidder's reporting strategy is bounded below by some linear function $\underline{r}(\cdot)$ whose slope is between $\frac{a}{a+n-1}$ and 1 , and with inverse $\underline{s}(\cdot)$ we will construct an upper bound $\bar{r}(\cdot)$.

First, observe that for any report $r_{j}$, no type greater than $\underline{s}\left(r_{j}\right)$ will report $r_{j}$. Suppose that under report profile $r$, type $s_{i}$ of bidder $i$ is pivotal at report $r_{j}$. Bidder $i$ 's payoff is

$$
a s_{i}+\sum s_{k}-a r_{j}-\sum_{k \neq i} r_{k}
$$

Since $s_{j} \leq \underline{s}\left(r_{j}\right)$, this payoff is no greater than

$$
a s_{i}+\underline{s}\left(r_{j}\right)-(a+1) r_{j}+(n-2) \max _{\hat{r}_{k} \leq r_{j}}\left(\underline{s}\left(\hat{r}_{k}\right)-\hat{r}_{k}\right)
$$

Since the slope of $\underline{s}(\cdot)$ is greater than 1 , the maximum is

$$
a s_{i}+(n-1) \underline{s}\left(r_{j}\right)-(a+n-1) r_{j}
$$

By assumption, $a>n-1$. Therefore, the slope in $r_{j}$ of the right hand expression is at most $\frac{(n-1)(a+n-1)}{a}-(a+n-1)$ which is negative. Letting $\bar{r}\left(s_{i}\right)$ be the $r_{j}$ for which this expression is zero, we can implicitly solve for $\bar{r}\left(s_{i}\right)$

$$
\bar{r}\left(s_{i}\right)=\frac{a s_{i}+(n-1) \underline{s}\left(\bar{r}\left(s_{i}\right)\right)}{a+n-1}
$$

and conclude that any report greater than $\bar{r}\left(s_{i}\right)$ is dominated for type $s_{i}$ by the report $\bar{r}\left(s_{i}\right)$.

Since $\underline{s}(\cdot)$ is linear, so will be $\bar{r}\left(s_{i}\right)$. And because the slope of $\underline{s}(\cdot)$ is greater than 1 , it follows that the slope of $\bar{r}\left(s_{i}\right)$ is also greater than 1 .

Now, we assume that all iteratively undominated reporting strategies are bounded above by some linear $\bar{r}(\cdot)$ with slope greater than 1 , with inverse $\bar{s}(\cdot)$, and derive a new lower bound.

Suppose that under report profile $r$, type $s_{i}$ of bidder $i$ is pivotal at report $r_{j}$. Bidder $i$ 's payoff is at least

$$
a s_{i}+\bar{s}\left(r_{j}\right)-(a+1) r_{j}+(n-2) \min _{\hat{r}_{k} \leq r_{j}}\left(\bar{s}\left(\hat{r}_{k}\right)-\hat{r}_{k}\right)
$$


Since the slope of $\bar{s}(\cdot)$ is less than 1 , the minimum is

$$
a s_{i}+(n-1) \bar{s}\left(r_{j}\right)-(a+n-1) r_{j}
$$

The slope in $r_{j}$ is negative, so we can set this expression equal to zero, solve for $\underline{r}\left(s_{i}\right)$ :

$$
\underline{r}\left(s_{i}\right)=\frac{a s_{i}+(n-1) \bar{s}\left(\underline{r}\left(s_{i}\right)\right)}{a+n-1}
$$

and conclude that any report less than $\underline{r}\left(s_{i}\right)$ is dominated for type $s_{i}$ by the report $\underline{r}\left(s_{i}\right)$. If the slope of $\bar{s}$ is greater than one, then the slope of $\underline{r}$ will also be greater than one.

We can use equations (11) and (12) to iteratively construct upper and lower bounds on the set of iteratively undominated reporting strategies. We now show that $\underline{r}(1)$ increases monotonically to 1 . Recall that in order for our construction of $\bar{r}(\cdot)$ to be valid, we required that the slope of $\underline{r}(\cdot)$ to be greater than $\frac{a}{a+n-1}$. Since this was satisfied by the initial lower bound, it will be satisfied by every subsequent lower bound provided the slopes increase along the sequence. Since each bound is linear, it suffices that the intercept $\underline{r}(1)$ increases monotonically. Furthermore, since the slope of $\underline{r}(\cdot)$ is less than one, it will follow that $\underline{r}(\cdot)$ increases monotonically to the identity mapping. Plugging in the identity mapping to equation 11 shows that the limit of $\bar{r}(\cdot)$ must also be the identity. Thus showing that $\underline{r}(1)$ increases to 1 will complete the proof.

Consider any value $0<\underline{r}(1)<1$. By linearity, the inverse is defined by $\underline{s}(r)=r / \underline{r}(1)$. Using equation (11), we can solve for $\bar{s}(r)$ :

$$
\bar{s}(r)=r\left[\frac{a+n-1-\frac{n-1}{\underline{r}(1)}}{a}\right]
$$

Plugging in to equation (12) and solving for $\underline{r}^{\prime}(1)$ (after some manipulation):

$$
\underline{r}^{\prime}(1)=\frac{a^{2}}{a^{2}+\left[\frac{1-\underline{r}(1)}{\underline{r}(1)}\right](n-1)^{2}}
$$

Since $\underline{r}(1)<1$ and $a>n-1, \underline{r}^{\prime}(1)>\underline{r}(1)$. This finishes the proof of the "if" part.

We now prove that the generalized VCG mechanism, for any arbitrary $f_{i}$ 's, is not dominance solvable if $a \leq n-1$. Consider the subset of reporting strategy 
profiles $\beta:=\prod \beta_{i}$ where $\beta_{i}=\left\{r_{i} \in R_{i}: r_{i}\left(s_{i}\right)=\alpha+\frac{a}{a+n-1} s_{i}, \alpha \in\left[0, \frac{n-1}{a+n-1}\right]\right\}$. We will show that for any reporting strategy $r_{i} \in \beta_{i}$ and alternative reporting strategy $r_{i}^{\prime} \in B_{i}$, there exists some reporting strategy $r_{-i} \in \beta_{-i}$ against which $r_{i}$ does strictly better than $r_{i}^{\prime}$ does. Hence $\beta$ must be a subset of any dominance solution, and hence there is no efficient dominance solution.

Fix any reporting strategy $r_{i} \in \beta_{i}$ and alternative reporting strategy $r_{i}^{\prime} \in B_{i}$. Since $r_{i}^{\prime} \neq r_{i}$, there exists $\hat{s}_{i} \in S_{i}$ such that $r_{i}^{\prime}\left(\hat{s}_{i}\right) \neq r_{i}\left(\hat{s}_{i}\right)$. Suppose $r_{i}\left(\hat{s}_{i}\right)<r<$ $r_{i}^{\prime}\left(\hat{s}_{i}\right)$. Consider $r_{j} \in \beta_{j}, j \neq i$, such that $r_{j}\left(s_{j}\right)=\alpha+\frac{a}{a+n-1} s_{j}$, where $\alpha=$ $\min \left\{r, \frac{n-1}{a+n-1}\right\}$. At the state of the world $s_{i}=\hat{s}_{i}$ and $\forall j \neq i, s_{j}=\frac{a+n-1}{a}(r-\alpha)$, employing reporting strategy $r_{i}$ results in bidder $i$ losing the object and getting net payoff $-f_{i}\left(r_{-j}\right)$. Whereas employing reporting strategy $r_{i}^{\prime}$ results in bidder $i$ winning the object and getting net payoff $a \hat{s}_{i}+(n-1) \frac{a+n-1}{a}(r-\alpha)-(a+$ $n-1) r-f_{i}\left(r_{-i}\right)$. If $r>\frac{n-1}{a+n-1}$, then $\alpha=\frac{n-1}{a+n-1}$, and the difference will be

$$
\begin{aligned}
& a \hat{s}_{i}+(n-1) \frac{a+n-1}{a}\left(r-\frac{n-1}{a+n-1}\right)-(a+n-1) r \\
= & a \hat{s}_{i}+(a+n-1) \frac{n-1-a}{a} r-\frac{(n-1)^{2}}{a} \\
< & a \hat{s}_{i}+(a+n-1) \frac{n-1-a}{a}-\frac{(n-1)^{2}}{a} \\
= & a \hat{s}_{i}-a \\
\leq & 0,
\end{aligned}
$$

where the strict inequality follows from $r<r_{i}^{\prime}\left(\hat{s}_{i}\right) \leq 1$. If $r \leq \frac{n-1}{a+n-1}$, then $\alpha=r$, and the difference will be

$$
\begin{aligned}
& a \hat{s}_{i}-(a+n-1) r \\
< & a \hat{s}_{i}-(a+n-1) r_{i}\left(\hat{s}_{i}\right) \\
\leq & a \hat{s}_{i}-(a+n-1) \frac{a}{a+n-1} \hat{s}_{i} \\
= & 0,
\end{aligned}
$$

where the strict inequality follows from the definition of $r$. So employing reporting strategy $r_{i}^{\prime}$ unambiguously results in lower net payoff at the state of the world $s_{i}=\hat{s}_{i}$ and $\forall j \neq i, s_{j}=\frac{a+n-1}{a}(r-\alpha)$. The case where $r_{i}^{\prime}\left(\hat{s}_{i}\right)<r<$ $r_{i}\left(\hat{s}_{i}\right)$ is handled symmetrically. Combining the two cases we conclude that there always exists some reporting strategies $r_{-i} \in \beta_{-i}$ against which $r_{i}$ does strictly better than $r_{i}^{\prime}$ does. This completes our proof. 
Let's summarize what we have and what we have not proved in this section. We have shown that, in the symmetric linear setting with $n \geq 3$ bidders, the generalized VCG mechanism is dominance solvable if and only if $a>n-1$. Since the generalized VCG mechanism is already the unique direct mechanism that implements the efficient allocation in ex post equilibrium, it is hopeless to construct other direct mechanisms that ex post dominance implement the efficient allocation when $a \leq n-1$. We summarize all these in the following corollary.

Corollary 5.2. In the symmetric linear setting with $n \geq 3$ bidders, there exists a direct mechanism that ex post dominance implements the efficient allocation if and only if $a>n-1$.

However, we have not proved that $a>n-1$ is necessary for ex post dominance implementation of the efficient allocation. The reason is that we have only looked at direct mechanisms. For most other equilibrium concepts, it suffices to look at direct mechanisms in order to obtain necessary conditions for implementation. This is because the revelation principle holds for most other equilibrium concepts. Unfortunately, the revelation principle breaks down for dominance solvable mechanisms. Since this is an observation that should be of independent interest, we shall discuss it in a separate section.

\section{FAILURE OF THE REVELATION PRINCIPLE}

We shall give an example in this section to demonstrate how the revelation principle fails to hold for dominance solution. Our example may not be the simplest one one can conceive, and makes use of a rather rich set of social alternatives. But it makes a point which we believe has not been addressed before in the literature. In the implementation literature, the revelation principle holds for most of the equilibrium concepts as long as we do not require unique implementation. However, when it comes to ex post dominance implementation (unique or not), the revelation principle breaks down.

Consider a situation with seven social alternatives $\left(x, y, w_{A}, w_{B}, z_{A}, z_{B}\right.$, and $o$ ), two players (1 and 2), and each player having two types $\left(s_{i}=i_{A}, i_{B}, i=\right.$ $1,2)$. The players have quasi-linear utilities, with the corresponding valuation functions being summarized by Table 1 . Table 1 also depicts the efficient rule, $f$, that the mechanism designer wants to implement. 


\begin{tabular}{|c|c|c|c|c|}
\hline$\left(s_{1}, s_{2}\right)=$ & $\left(1_{A}, 2_{A}\right)$ & $\left(1_{A}, 2_{B}\right)$ & $\left(1_{B}, 2_{A}\right)$ & $\left(1_{B}, 2_{B}\right)$ \\
\hline$v_{1}(x)=v_{2}(x)=$ & 2 & 0 & 0 & 2 \\
\hline$v_{1}(y)=v_{2}(y)=$ & 0 & 2 & 2 & 0 \\
\hline$v_{1}\left(w_{A}\right)=$ & 0 & 3 & 0 & 3 \\
\hline$v_{1}\left(w_{B}\right)=$ & 3 & 0 & 3 & 0 \\
\hline$v_{1}\left(z_{A}\right)=v_{1}\left(z_{B}\right)=v_{1}(o)=$ & 0 & 0 & 0 & 0 \\
\hline$v_{2}\left(z_{A}\right)=$ & 0 & 0 & 3 & 3 \\
\hline$v_{2}\left(z_{B}\right)=$ & 3 & 3 & 0 & 0 \\
\hline$v_{2}\left(w_{A}\right)=v_{2}\left(w_{B}\right)=v_{2}(o)=$ & 0 & 0 & 0 & 0 \\
\hline$f\left(s_{1}, s_{2}\right)=$ & $x$ & $y$ & $y$ & $x$ \\
\hline
\end{tabular}

Table 1: Players' valuations and the efficient rule.

We first show that no dominance solvable direct mechanisms can (truthfully) implement $f$. Suppose an implementing direct mechanism exists. Such a direct mechanism will be fully characterized by a pair of transfer functions, $t_{1}\left(s_{1}, s_{2}\right)$ and $t_{2}\left(s_{1}, s_{2}\right)$.

If truth-telling is the dominance solution, there must be some untruthful strategy that is dominated for some player, say player 1. An untruthful strategy is dominated for player 1 only if there is some type, say $1_{A}$ for player 1 such that telling the truth (reporting $A$ ), is at least as good as lying (reporting $B$ ), for every type and report of player 2 . This yields the following set of inequalities, with at least one strict.

$$
\begin{aligned}
& v_{1}\left(f\left(1_{A}, 2_{A}\right) \mid 1_{A}, 2_{A}\right)+t_{1}\left(1_{A}, 2_{A}\right) \geq v_{1}\left(f\left(1_{B}, 2_{A}\right) \mid 1_{A}, 2_{A}\right)+t_{1}\left(1_{B}, 2_{A}\right) \\
& v_{1}\left(f\left(1_{A}, 2_{B}\right) \mid 1_{A}, 2_{A}\right)+t_{1}\left(1_{A}, 2_{B}\right) \geq v_{1}\left(f\left(1_{B}, 2_{B}\right) \mid 1_{A}, 2_{A}\right)+t_{1}\left(1_{B}, 2_{B}\right) \\
& v_{1}\left(f\left(1_{A}, 2_{A}\right) \mid 1_{A}, 2_{B}\right)+t_{1}\left(1_{A}, 2_{A}\right) \geq v_{1}\left(f\left(1_{B}, 2_{A}\right) \mid 1_{A}, 2_{B}\right)+t_{1}\left(1_{B}, 2_{A}\right) \\
& v_{1}\left(f\left(1_{A}, 2_{B}\right) \mid 1_{A}, 2_{B}\right)+t_{1}\left(1_{A}, 2_{B}\right) \geq v_{1}\left(f\left(1_{B}, 2_{B}\right) \mid 1_{A}, 2_{B}\right)+t_{1}\left(1_{B}, 2_{B}\right) .
\end{aligned}
$$

Using the valuations from table $1,(14)$ and (15) reduce to

$$
\begin{aligned}
& t_{1}\left(1_{A}, 2_{A}\right) \geq 2+t_{1}\left(1_{B}, 2_{A}\right) \\
& t_{1}\left(1_{A}, 2_{B}\right) \geq 2+t_{1}\left(1_{B}, 2_{B}\right) .
\end{aligned}
$$

Since truth-telling is a dominance solution, it is also an ex post equilibrium in this finite game. Therefore truthful reporting should be a best-response for 1 to truthful reporting by 2 for every type profile, in particular whenever player 
1 is of type $1_{B}$ :

$$
\begin{aligned}
& v_{1}\left(f\left(1_{B}, 2_{A}\right) \mid 1_{B}, 2_{A}\right)+t_{1}\left(1_{B}, 2_{A}\right) \geq v_{1}\left(f\left(1_{A}, 2_{A}\right) \mid 1_{B}, 2_{A}\right)+t_{1}\left(1_{A}, 2_{A}\right) \\
& v_{1}\left(f\left(1_{B}, 2_{B}\right) \mid 1_{B}, 2_{B}\right)+t_{1}\left(1_{B}, 2_{B}\right) \geq v_{1}\left(f\left(1_{A}, 2_{B}\right) \mid 1_{B}, 2_{B}\right)+t_{1}\left(1_{A}, 2_{B}\right) .
\end{aligned}
$$

This can be rewritten as

$$
\begin{aligned}
& t_{1}\left(1_{A}, 2_{A}\right) \leq 2+t_{1}\left(1_{B}, 2_{A}\right) \\
& t_{1}\left(1_{A}, 2_{B}\right) \leq 2+t_{1}\left(1_{B}, 2_{B}\right) .
\end{aligned}
$$

In view of (17)-(18), inequalities (19) and (20) are satisfied with equality implying that player 1 is indifferent between reporting $A$ or $B$ when type $1_{B}$ and 2 tells the truth.

Notice that (17) and (18) also imply

$$
\begin{aligned}
& 2+t_{1}\left(1_{A}, 2_{A}\right) \geq t_{1}\left(1_{B}, 2_{A}\right) \\
& 2+t_{1}\left(1_{A}, 2_{B}\right) \geq t_{1}\left(1_{B}, 2_{B}\right)
\end{aligned}
$$

so that

$$
\begin{aligned}
& v_{1}\left(f\left(1_{A}, 2_{A} \mid 1_{B}, 2_{B}\right)+t_{1}\left(1_{A}, 2_{A}\right) \geq t_{1}\left(1_{B}, 2_{A}\right)\right. \\
& v_{1}\left(f\left(1_{A}, 2_{B} \mid 1_{B}, 2_{A}\right)+t_{1}\left(1_{A}, 2_{B}\right) \geq t_{1}\left(1_{B}, 2_{B}\right)\right.
\end{aligned}
$$

i.e. 1 is willing to report $A$ when he is type $1_{B}$ and 2 is lying.

We have shown that 1 is willing to report $A$ for every type profile and for every behavior of player 2 . Moreover, at least one of the preferences is strict (in (13)-(16)). Therefore, the strategy of reporting $A$ independent of type is an ex post weakly dominant strategy for 1 and hence could never be deleted. But then truthful reporting could not be the dominance solution.

Now we demonstrate that $f$ can be ex post dominance implemented with an indirect mechanism, and hence the revelation principle fails. The indirect mechanism has message space $\left\{i_{A}, i_{B}, i_{C}\right\}$ for each player $i=1,2$ ( $i_{C}$ can be interpreted as a "fictitious type" that is augmented to the message space of the direct mechanism), and transfer functions identical to zero. The outcome function is depicted in Figure 3.

The process of iterative elimination of dominated strategies goes as follows. In the first round, for any player $i=1,2$, when his true type is $i_{A}$, reporting $i_{B}$ is dominated by reporting $i_{C}$; similarly, when his true type is $i_{B}$, reporting 


\begin{tabular}{|c|c|c|c|}
\hline & $" 2_{A} "$ & $" 2_{B} "$ & $" 2_{C} "$ \\
\hline$" 1_{A} "$ & 0 & $z_{B}$ & $z_{A}$ \\
\hline$" 1_{B} "$ & $w_{B}$ & $x$ & $y$ \\
\hline$" 1_{C} "$ & $w_{A}$ & $y$ & $x$ \\
\hline
\end{tabular}

Figure 3: A dominance solvable indirect mechanism.

$i_{A}$ is dominated by reporting $i_{C}$. In the second round, for any player $i=1,2$, reporting $i_{C}$ is dominated no matter what his true type is. So after two rounds of elimination, only truth-telling survives. We urge the reader to do the straightforward verification that truth-telling is an ex post equilibrium both for this indirect mechanism and for its corresponding direct mechanism (i.e., the direct mechanism derived by eliminating messages not used on the equilibrium path).

\section{References}

Ausubel, L. M. (1999). A generalized vickrey auction. Mimeo, University of Maryland.

Bergemann, D., \& Välimäki, J. (2002). Information acquisition and efficient mechanism design. Econometrica, 70(3), 1007-1033.

Dasgupta, P., \& Maskin, E. (2000). Efficient auctions. Quarterly Journal of Economics, 115(2), 341-388.

Harstad, R. M., \& Levin, D. (1985). A class of dominance solvable common-value auctions. Review of Economic Studies, 52(3), 525-528.

Jehiel, P., \& Moldovanu, B. (2001). Efficient design with interdependent valuations. Econometrica, 69(5), 1237-1259.

Krishna, V. (2003). Asymmetric english auctions. Journal of Economic Theory, 112(2), 261-288.

Maskin, E. (1992). Auctions and privatization. In H. Siebert (Ed.), Privatization: Symposium in honour of Hebert Giersch (pp. 115-136). Tübingen: J.C.B. Mohr. 
Perry, M., \& Reny, P. J. (1999). An ex-post efficient auction. Discussion Paper \#200, Center for Rationality and Interactive Decision Theory, Hebrew University of Jerusalem. 